\title{
Dentate Gyrus Sharp Waves, a Local Field Potential Correlate of Learning in the Dentate Gyrus of Mice
}

\author{
Kolja Meier, ${ }^{1,2}$ Andrea Merseburg, ${ }^{2,3}{ }^{\oplus}$ Dirk Isbrandt, ${ }^{2,3 *}$ Stephan Lawrence Marguet, ${ }^{2,3 *}$ and ${ }^{\circledR}$ Fabio Morellini ${ }^{1 \star}$ \\ ${ }^{1}$ Behavioral Biology, Center for Molecular Neurobiology, University Medical Center Hamburg-Eppendorf, Hamburg 20251, Germany, ${ }^{2}$ German \\ Center for Neurodegenerative Diseases (DZNE), Bonn 53175, Germany, and ${ }^{3}$ Institute for Molecular and Behavioral Neuroscience, Faculty of \\ Medicine, University of Cologne, Cologne 50937, Germany
}

The hippocampus plays an essential role in learning. Each of the three major hippocampal subfields, dentate gyrus (DG), $\mathrm{CA} 3$, and CA1, has a unique function in memory formation and consolidation, and also exhibit distinct local field potential (LFP) signatures during memory consolidation processes in non-rapid eye movement (NREM) sleep. The classic LFP events of the CA1 region, sharp-wave ripples (SWRs), are induced by CA3 activity and considered to be an electrophysiological biomarker for episodic memory. In LFP recordings along the dorsal CA1-DG axis from sleeping male mice, we detected and classified two types of LFP events in the DG: high-amplitude dentate spikes (DSs), and a novel event type whose current source density (CSD) signature resembled that seen during CA1 SWR, but which, most often, occurred independently of them. Because we hypothesize that this event type is similarly induced by CA3 activity, we refer to it as dentate sharp wave (DSW). We show that both DSWs and DSs differentially modulate the electrophysiological properties of SWR and multiunit activity (MUA). Following two hippocampus-dependent memory tasks, DSW occurrence rates, ripple frequencies, and ripple and sharp wave (SW) amplitudes were increased in both, while SWR occurrence rates in dorsal CA1 increased only after the spatial task. Our results suggest that DSWs, like SWRs, are induced by CA3 activity and that DSWs complement SWRs as a hippocampal LFP biomarker of memory consolidation.

Key words: dentate gyrus; dentate sharp wave; dentate spike; hippocampus; sharp-wave ripple

Significance Statement

Awake experience is consolidated into long-term memories during sleep. Memory consolidation crucially depends on sharpwave ripples (SWRs), which are local field potential (LFP) patterns in hippocampal CA1 that increase after learning. The dentate gyrus (DG) plays a central role in the process of memory formation, prompting us to cluster sharp waves (SWs) in the DG [dentate SWs (DSWs)] during sleep. We show that both DSW coupling to CA1 SWRs, and their occurrence rates, robustly increase after learning trials. Our results suggest that the DG is directly affected by memory consolidation processes. DSWs may thus complement SWRs as a sensitive electrophysiological biomarker of memory consolidation in mice.

\section{Introduction}

The hippocampus is recognized as one of the core brain structures responsible for cognitive functions in humans and other mammals. The three major hippocampal subfields: dentate gyrus (DG), CA3, and CA1 each play distinct roles in the processing of

\footnotetext{
Received Aug. 1, 2019; revised Mar. 27, 2020; accepted June 9, 2020.

Author contributions: D.I. and F.M. designed research; K.M. and A.M. performed research; K.M., S.L.M., and F.M. analyzed data; K.M., D.I., and F.M. wrote the paper.

*D.I., S.L.M, and F.M. contributed equally to this work.

This work was supported by Deutsche Forschungsgemeinschaft (DFG) Grants SFB936, B3 (to F.M. and D.I.), SFB1089, A5 (to D.I. and S.L.M.), and IS63/3-1/2 (to D.I.). We thank V. Kraus and Ricardo Melo Neves for behavioral scoring and A. Sirota (Ludwig Maximilian University, Munich, Germany) for comments on the manuscript.

The authors declare no competing financial interests.

Correspondence should be addressed to Dirk Isbrandt at dirk.isbrandt@dzne.de.

https://doi.org/10.1523/JNEUROSCI.2275-19.2020

Copyright $\odot 2020$ the authors
}

information relevant for learning and memory. For instance, the DG is likely to be relevant for pattern separation during information processing, whereas pattern completion may require computational processes supported by the CA3 subregion (Treves and Rolls, 1992; O'Reilly and McClelland, 1994; Nakazawa et al., 2002; Leutgeb et al., 2007). The hippocampus is not only involved in learning and memory formation but also plays a role in long-term consolidation of newly generated memories, a process that partially depends on proper brain activity during the first sleep period after a learning experience (Wilson and McNaughton, 1994; Lee and Wilson, 2002; Perrin et al., 2004; Girardeau et al., 2009; Maingret et al., 2016). Hippocampal and cortical activity during sleep in rodents comprises two stages that resemble rapid eye movement (REM) sleep and non-REM (NREM) sleep described in humans (Stickgold, 2005; Abel et al., 2013). The rodent REM-like sleep is characterized by $\theta / \gamma$ oscillations, whereas neocortical slow oscillations and sleep spindles 
co-occur with high-frequency CA1 sharp-wave ripples (SWRs) during NREM sleep, also called slow-wave sleep (SWS, Buzsáki, 1986; Ylinen et al., 1995; Buzsáki et al., 2003; Girardeau et al., 2009). Several studies in rodents and humans suggest that new memories are reactivated and consolidated during SWRs (Wilson and McNaughton, 1994; Girardeau et al., 2009; Zhang et al., 2018). In the context of learning and memory, it is noteworthy that SWRs in CA1 are induced by spontaneous population bursts of CA3 pyramidal cells, and that suppression of SWRs impairs rodents' performance in a hippocampus-dependent memory task (Girardeau et al., 2009).

CA3 pyramidal neurons not only project in a feed-forward manner via Schaffer collaterals to CA1, but also send both feed-back activation and inhibition to the DG: excitatory projections to dentate granule cells (DGCs) and hilar mossy cells (Scharfman, 2007; Shi et al., 2019), and inhibitory interneuron projections from CA1 and CA3 to DGCs (Szabo et al., 2017). Activation of the excitatory backprojections from CA3 results in sharp waves (SWs) with current sinks in the DG inner molecular layer (IML; Ylinen et al., 1995; Penttonen et al., 1997; Sullivan et al., 2011). As a result, SWR-associated neuronal activity is not restricted to the CA1 region, but can also be observed in hilar mossy and DGCs in acute hippocampal slices (Swaminathan et al., 2018) and dentate interneurons (Szabo et al., 2017). During SWS and awake immobility, entorhinal cortical (EC) inputs to the middle molecular layer (MML) and outer molecular layer (OML) of the DG induce short-lasting and large-amplitude field potentials called dentate spikes (DSs) that suppress SWR generation (Bragin et al., 1995; Penttonen et al., 1997), thereby potentially modulating memory consolidation. Stimulation of the ventral hippocampal commissure during DS was also shown to be sufficient to impair associative learning (Nokia et al., 2017).

Several studies were designed to use SWR or DS-triggered stimulation of the ventral hippocampus ( $\mathrm{vHC}$ ) to investigate the role of SWRs or DSs in memory consolidation (Girardeau et al., 2009; Nokia et al., 2017; Lensu et al., 2019). There are only a few studies on DG feed-back activation and, to date, none of them has addressed its role in memory consolidation processes (Penttonen et al., 1997; Swaminathan et al., 2018). Here, we hypothesize that activation of the DG correlates with CA1 SWR activity and that awake experience has an effect on DG LFP patterns. Using chronic in vivo LFP depth profile recordings along the dorsal CA1-DG axis in mice, we automatically detected and classified spontaneous DG LFP activity during SWS before and after subjecting the mice to hippocampus-dependent learning tasks. We observed a class of DG events, the dentate SW (DSW), that differed from DSs by a current source in the ML, which were occasionally synchronous to CA1 SWRs and displayed increased coupling to SWRs and increased occurrence rates after learning. We, therefore, propose DSWs may be a sensitive indicator of hippocampus-dependent learning and complement locally detected CA1 SWRs in mice.

\section{Materials and Methods}

\section{Animals}

Male C57BL/6J mice (25-36 g) were transferred from a specific pathogen-free breeding facility of the University Medical Center HamburgEppendorf into a vivarium with an inverted 12/12 h light/dark cycle (light off at 7 A.M.) and kept under standard housing conditions $\left(23 \pm 1^{\circ} \mathrm{C}, 40-60 \%\right.$ humidity, food and water ad libitum). After two weeks of acclimatization, mice underwent the experiments that were performed between 9 A.M. and 7 P.M. under red light. Mice were aged between six and nine months during the experiments. All experimental procedures were approved by the Ministry of Science and Public Health of the City State of Hamburg, Germany.

\section{Implantation of recording electrodes}

Mice were anesthetized with 3.5-4\% isoflurane in 100\% oxygen and kept at $0.8-1.5 \%$ isoflurane throughout the surgery. For analgesia, mice received a subcutaneous injection of $0.025-0.05 \mathrm{mg} / \mathrm{kg}$ buprenorphine (Temgesic), $5.0 \mathrm{mg} / \mathrm{kg}$ carprofen (Rimadyl), and a single dose of $7.5 \mathrm{mg} /$ $\mathrm{kg}$ of the antibiotic enrofloxacin (Baytril). Body temperature was monitored and kept constant at $36.5^{\circ}$ with a homeothermic heating pad (Stoelting). The head was placed in a stereotaxic frame (Kopf Instruments), the skull was exposed, and a small hole was drilled into the skull above the hippocampus $1.7-2.0 \mathrm{~mm}$ posterior to bregma and 1.5$1.6 \mathrm{~mm}$ right from the midline (Paxinos and Franklin, 2007). Two wires, each soldered to a stainless-steel screw (Small Parts), were implanted $\sim 1.0 \mathrm{~mm}$ posterior from $\lambda$ and $\sim 1.0 \mathrm{~mm}$ left or right of the midline above the cerebellum and used for ground or reference, respectively. A 16-site linear silicon probe with $100-\mu \mathrm{m}$ spacing (A1x16-3 mm-100177-CM16LP or A1x16-5 mm-100-177-CM16, NeuroNexus) was lowered vertically into the dorsal hippocampus ( $\mathrm{dHC}$ ) along the CA1-DG axis (2.0-2.2 $\mathrm{mm}$ in depth) and fixed to the skull by dental cement. A copper mesh was used to surround and protect the implant, attached to the skull by dental cement, and then connected to animal ground for electrical shielding. Mice were allowed to recover for at least $4 \mathrm{~d}$.

\section{Data acquisition}

Amplifying headstages [16 channel $2 \times$ gain, T16G2 (TBSI)] were attached to the silicon probe and connected to the Digital Lynx 10S data acquisition system (Neuralynx). Data were digitally filtered (0.5- to 9000 $\mathrm{Hz}$ bandpass) and digitized as 16-bit integers with a sampling rate of $32,552 \mathrm{~Hz}$. Two LEDs were attached to the headstage and used to monitor the position of the mouse by simultaneous video recording. For LFP analysis, the $32,552 \mathrm{~Hz}$ broadband signal was downsampled to $1252 \mathrm{~Hz}$. Current source densities (CSDs) were computed from the LFP using spline iCSD (Pettersen et al., 2006) with a conductivity of $0.3 \mathrm{~S} / \mathrm{m}$, a source diameter of $0.5 \mathrm{~mm}$, and no post hoc smoothing. All in vivo data were analyzed and visualized in MATLAB (The MathWorks) or Neuroscope (Hazan et al., 2006; http://neurosuite.sourceforge.net/). Sleep and wake states were manually scored by the characteristic spectrogram of behavioral states (Buzsáki et al., 2003) and movement, as determined from the simultaneously-recorded, synchronized video.

\section{Electrode position}

Histological procedures

Animals were deeply anesthetized via intraperitoneal injection of $1 \mu \mathrm{l} / \mathrm{g}$ body weight anesthetic (12\% Ketanest, $8 \%$ Sedaxylan in $0.9 \% \mathrm{NaCl}$ solution). A thoracotomy was performed, and the right atrium was perforated. The mouse was then perfused with $150 \mathrm{ml}$ PBS into the left ventricle. For tissue fixation, the mouse was subsequently perfused with $50 \mathrm{ml}$ of $4 \%$ paraformaldehyde (PFA; Roti Histofix, Roth). Mice were then decapitated and the brain was removed, fixed overnight in $4 \%$ PFA at $4^{\circ} \mathrm{C}$, and stored in PBS at $4^{\circ} \mathrm{C}$. For vibratome sections, brains were glued to a socket, submerged in PBS, and 50- or 100- $\mu$ m sagittal sections were cut using a vibratome (VT1000S, Leica). Brain sections were investigated with a Zeiss AX10 microscope using an AxioCam MRC color camera and the corresponding software AxioVision (Zeiss).

\section{Data analysis}

Hippocampal layer identification

CA1 stratum pyramidale (CA1p) was identified by the largest ripple oscillation $(100-250 \mathrm{~Hz})$ amplitude in the LFP. The CA1 stratum radiatum was identified by the largest SW amplitude in the CSD during ripple oscillations detected in CA1. DG analysis was restricted to the dorsal blade (DG channels). The DG granule cell layer (DG-GCL) was identified by the occurrence of DSs (Bragin et al., 1995). Following our histology and the Allen mouse brain reference atlas (Allen Institute for Brain Science, 2004; Lein et al., 2007), DG molecular layer (DG-ML) channels were a priori determined as the two channels dorsal (i.e., 100 and $200 \mu \mathrm{m}$ ) to the DG-GCL. The estimated DG inner molecular layer (DG- 
IML) was the channel next to the DG-GCL (i.e., $100-\mu \mathrm{m}$ distance), and the DG outer molecular layer (DG-OML) was identified as the channel $200 \mu \mathrm{m}$ distant to the DG-GCL. It is important to note that the DG-ML is a three-layered structure consisting of OML, MML, and IML. Because of the $100-\mu \mathrm{m}$ distance between the electrode sites, we could not reliably distinguish between MML and OML. Therefore, we labeled MML/OML electrode sites as OML. The border between DG-ML and CA1 was verified in two ways. (1) We analyzed the average waveforms of DG-GCL, triggered by current sinks exceeding 4 SDs of the bandpass-filtered (1$100 \mathrm{~Hz}$ ) CSD amplitude in CA1 and DG-ML channels. In line with previous studies on DS (Bragin et al., 1995), large amplitude current sinks in the DG-ML are usually accompanied by a large amplitude current source in DG-GCL. (2) We analyzed the temporal organization of slow $(30-80 \mathrm{~Hz})$ and fast $(80-150 \mathrm{~Hz}) \gamma$ oscillation amplitudes in CA1 and DG channels during REM sleep. CA1 and DG $\gamma$ oscillations showed different phase relationships to the CA1p LFP $\theta$ phase. The shift of this phase relationship can be observed at the CA1-DG border (Lasztóczi and Klausberger, 2017).

\section{Ripple detection}

Both the detection of ripple oscillations in CA1p and calculation of ripple properties (amplitude, frequency, duration) were conducted using the Freely Moving Animal Toolbox (FMAtoolbox, M. Zugaro; http:// fmatoolbox.sourceforge.net/).

\section{Dentate wave detection and clustering}

We used the bandpass-filtered $(1-150 \mathrm{~Hz})$ CSD of the DG-GCL, DGIML, and DG-OML for the detection and clustering of dentate waves and calculated the sum of the rectified z-scored amplitudes of the DGGCL, DG-IML, and DG-OML CSD channels (referred to as S-DGAmp). We used the z-scored amplitudes to avoid a bias toward channels with the highest absolute amplitude. Peaks of the S-DG-Amp signal exceeding a threshold of three SD with a minimum half-peak width of $6.4 \mathrm{~ms}$ (i.e., eight samples of the $1252 \mathrm{~Hz}$ signal) were used for further analysis. The minimum interval between peaks was $100 \mathrm{~ms}$. We then detected local maxima in the CSD of the DG channels in temporal windows around dentate waves in which the S-DG-Amp exceeded 1.5 SD, which yielded amplitudes for the individual CSD signals (i.e., DG-GCL, DG-IML, DG-OML). We defined the amplitude of the local maxima of the respective CSD signals by their peak prominence. Peak prominence (as calculated by the MATLAB function findpeaks) is defined as a local maxima's height relative to the tallest of the furthest adjacent minima that enclose no taller maxima. "A low isolated peak can be more prominent than one that is higher but is an otherwise unremarkable member of a tall range" (MATLAB and Signal Processing Toolbox Release 2019a, The MathWorks). We refer to the aligned amplitude profile as the peak prominence profile (PPP). We used a t-distributed stochastic neighbor embedding (t-SNE) implementation for MATLAB (van der Maaten, 2014) for dimensionality reduction of the PPP. The t-SNE map of the PPP was clustered with the DBSCAN (Ester et al., 1996) algorithm, with constant minimum core points (minPts) and decreasing neighborhood distance $(\varepsilon)$, until clusters in the t-SNE map were evident and separated from each other. We manually labeled the identified clusters, inspired by an existing method for clustering of spike data (Dimitriadis et al., 2018). The resulting clusters were labeled as DS2, DSW, or Other by analysis of the sink-source distribution in the PPP.

Wavelets (Gabor family) or multitaper spectrograms (computed using Chronux; http://chronux.org/; Bokil et al., 2010) of original and whitened LFP signals were used for visualization.

Unit detection was performed using the NDmanager suite (Hazan et al., 2006). Units were detected in the CA1p, the DG-GCL, and in the respective neighboring channels. To exclude noise from our analysis, we clustered the units in the respective channel groups (i.e., CAlp with neighboring channels, DG-GCL with neighboring channels) using KlustaKwik 2.0 (Kadir et al., 2014). After discarding artifacts, we only analyzed those clusters in which spike waveforms were largest on the CA1p or DG-GCL channels, merging them into CAlp MUA or DG MUA, respectively.

Event (SWR, DSW, DS2)/MUA cross-correlograms (CCGs) were normalized by the product of both firing rates so that the CCGs would asymptote to 1 and yielded the ratio of coincidences above that expected for two uncorrelated spike trains.

\section{Behavioral tests}

Cued fear conditioning

In the cued fear conditioning test, mice had to learn the association between the unconditioned stimulus (US; an electric foot shock) and the conditioned stimulus (CS, $20 \mathrm{~s}$ light). Mice were conditioned in a dark box $(23.5 \times 23.5 \mathrm{~cm}$ and $19.5 \mathrm{~cm}$ high, illumination $=0$ lux $)$ with Plexiglas walls and ceiling and a stainless grid floor connected to an electric shock generator. Mice were placed in the box for $720 \mathrm{~s}$. The box was then illuminated for $20 \mathrm{~s}$ by white light at $360,420,480,540,600$, and $660 \mathrm{~s}$. During the last second of each light period, mice received an electric foot shock $(0.25 \mathrm{~mA}, 1 \mathrm{~s})$. At $720 \mathrm{~s}$, the mouse was placed back in the home cage. Twenty-four hours after conditioning, mice were re-exposed to the context for $25 \mathrm{~min}$. The chamber was illuminated with the same light source used as the CS from 360 to $1500 \mathrm{~s}$ in 60 -s intervals for $20 \mathrm{~s}$ (i.e., from 360 to $380 \mathrm{~s}$, from 420 to $440 \mathrm{~s}$, etc.). The conditioned response was manually analyzed by quantifying the percentage of time spent freezing (defined as the absence of body movements) using the software The Observer (Noldus). We recorded LFPs during sleeping phases in the home cage before and after the conditioning trial.

\section{Spatial learning}

Spatial learning was assessed via an ecologically relevant learning paradigm (Fellini and Morellini, 2013). Male mice were placed in an open arena for $30 \mathrm{~min}$. The arena was a white, laminated, square, wooden plate surrounded by transparent Plexiglas walls of $10 \mathrm{~cm}$ in height $(79.5$ $\times 79.5 \mathrm{~cm})$. Transparent Plexiglas walls $(26 \mathrm{~cm}$ high) were added to three of the four corners to create three boxes $(14 \times 14 \mathrm{~cm})$, and two to three cohabitating female mice were placed in one of them during the exposure trials. The walls had holes at the bottom to allow the male mouse in the arena to interact with the female mice in the corner. We conducted one exposure trial, followed by one short-term memory recall trial (in an arena absent of females) and two further exposure trials on day one. All trials lasted for $30 \mathrm{~min}$. Mice were placed back in their home cage at the end of each trial. The minimum intertrial interval was $60 \mathrm{~min}$. LFP during cage behavior was recorded before, between (while mice were in their home cage), and after the trials. Twenty-four hours after the learning trials, mice underwent a test trial to analyze long-term memory retention. Male mice were placed in an arena in the absence of female mice and allowed to move for $30 \mathrm{~min}$. To avoid the presence of olfactory cues related to the female mice, the male mice were placed in an apparatus strictly identical to the one used during the learning trials, with the sole difference that no female mouse had ever entered it. The time spent in the proximity of the females' box compared with the other two boxes (defined as when the head of the mouse was at a distance of $\leq 3 \mathrm{~cm}$ from the walls of the boxes) was measured with the software Ethovision (Noldus).

\section{Experimental design and statistical analyses}

For ease of reading, the data given in brackets in the text refers to mean $\pm \mathrm{SD}$ if not stated otherwise. Data were analyzed with ANOVA for repeated measurements with type of event (e.g., DSW and DS2) and time point (e.g., different sleep phases or different temporal relationship between the analyzed co-occurring events) as within-groups factors, followed by Turkey's HSD post hoc analyses when appropriate (i.e., the presence of a significant effect of interaction between factors). All tests were two-tailed and the level of significance was set at $p<0.05$.

\section{Results}

\section{Hippocampal depth profile recordings reveal distinct LFP patterns}

To characterize the spontaneous DG network activity during sleep, adult mice were implanted with 16-channel linear silicon probes in the $\mathrm{dHC}$ along the CA1-DG axis. Using probes with $100-\mu \mathrm{m}$ spacing between the electrode sites enabled us to record a depth profile spanning the CA1 pyramidal layer (CA1p) to the 

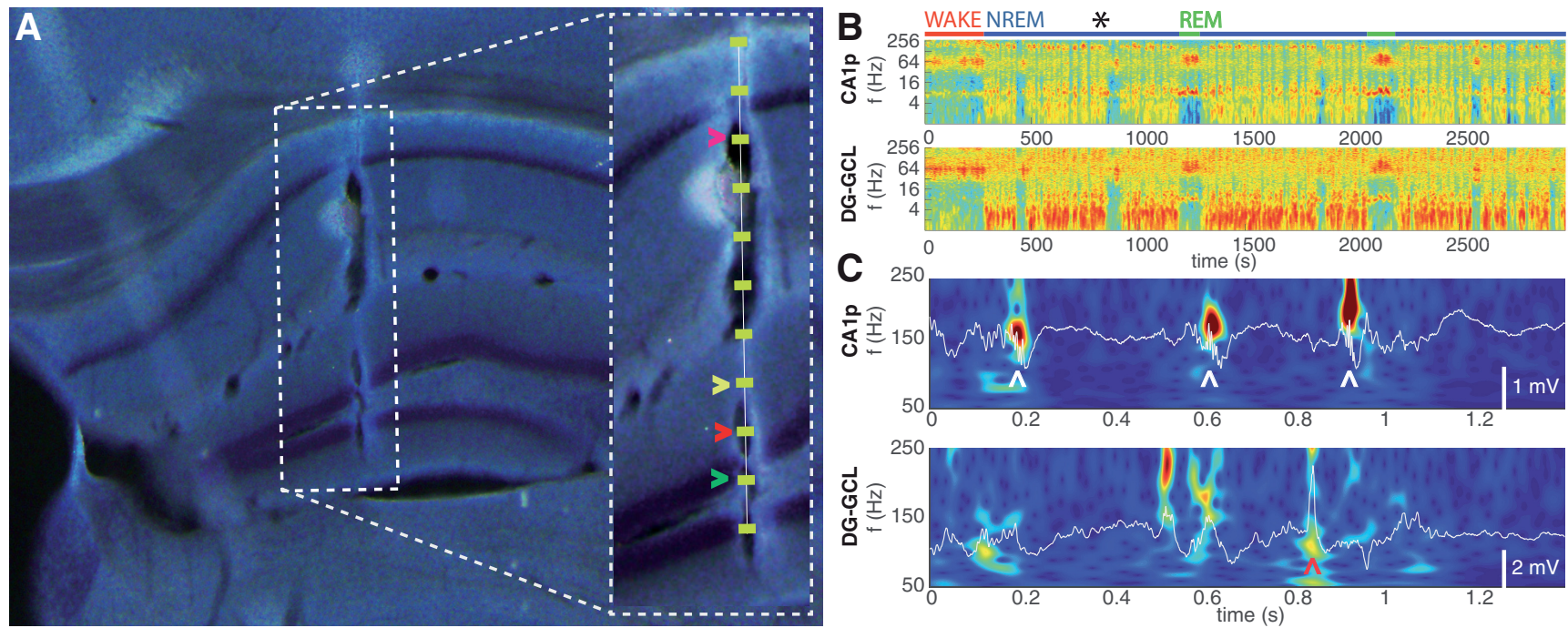

Sharp-wave ripple (SWR)

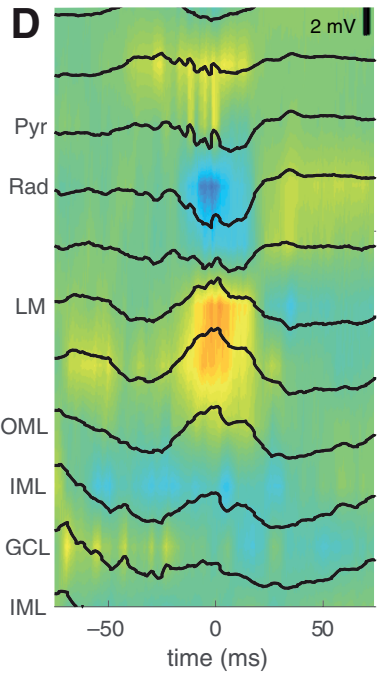

Dentate spike type 2 (DS2)

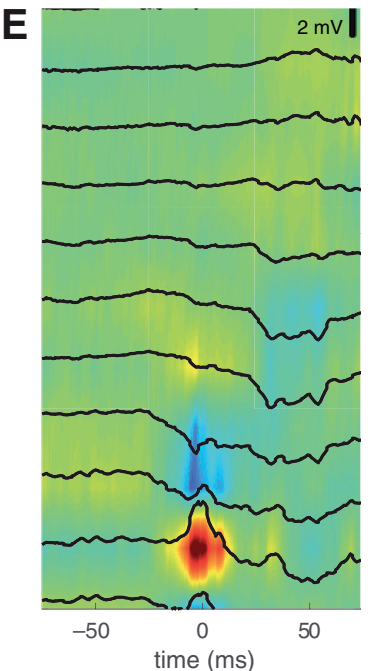

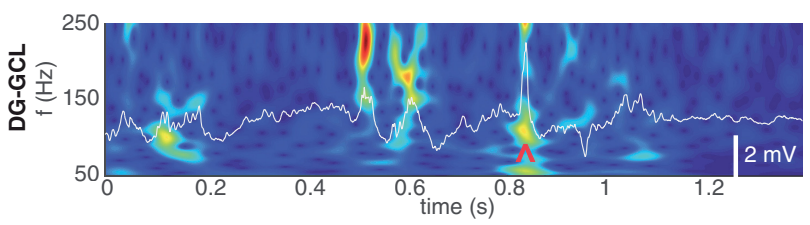

Dentate sharp wave (DSW)

$F$

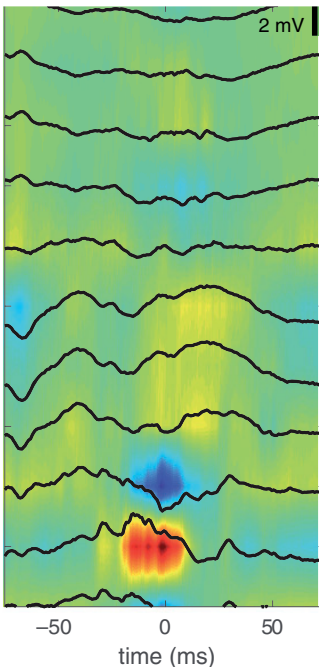

SWR with DSW

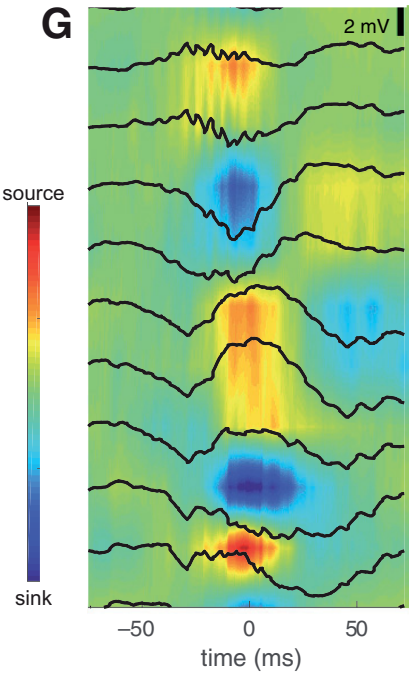

Figure 1. Depth profile recordings using linear silicon probes during sleep reveal classic and novel LFP patterns. $A$, Histologic identification of the silicon probe position in the dHC of a chronically implanted mouse. The white dashed square indicates the zoomed area shown in the inset, which highlights the deduced positions of the 16 electrode sites with $100-\mu \mathrm{m}$ spacing as yellow rectangles. Arrows indicate the anatomical location of specific recording sites: CA1p (magenta), DG-OML (yellow), DG-IML (red), DG-GCL (green). B, Characteristic spontaneous time-frequency spectrogram during awake, NREM, or REM sleep periods in the CA1p and DG-GCL. The asterisk $\left(^{*}\right)$ indicates the time point expanded in $\boldsymbol{C}$. Frequency on the $y$-axis is log-scaled. $C$, Detailed view of a NREM sleep period: high-frequency band spectrogram of the CA1p (top) and DG-GCL (bottom) with LFP (white) superimposed. White upward arrows on the CA1p spectrogram indicate ripple oscillations. Magenta upward arrow on the DG-GCL trace indicates a DS. D-G, Examples of spontaneous characteristic depth profiles activity of the CA1-DG axes as CSD plots with the LFP traces superimposed. Morphologic relationship to CA1 pyramidal neurons (magenta) and DG granule cells (green) is indicated on the left. The events shown were identified as follows: SWR $(\boldsymbol{D})$, DS2 $(\boldsymbol{E})$, a single DSW $(\boldsymbol{F})$, and a DSW coinciding with a SWR in CA1 (G). Note that a DS2 shows a sink in the OML, while a DSW shows a source in the 0ML. The DS2 further appears as a sharper potential in the CSD and LFP with shorter duration and larger slope. CSD and LFP scaling in $\mathbf{D}-\mathbf{G}$ is identical; the examples shown refer to the same mouse.

DG hilus. For baseline characterization, we analyzed in total 879 min of baseline NREM sleep obtained from seven mice on 12 recording days (range per mouse $79-212 \mathrm{~min}$ ). Electrode positions were verified using spontaneous, characteristic LFP patterns during REM and NREM sleep in combination with the histological identification of the silicon probe position (Fig. 1A; for details, see Materials and Methods). Using characteristic spectral properties in the CAlp and DG in combination with animal movement, we identified periods of sleep and wakefulness (Fig. 1B). During the NREM sleep periods, CA1p showed prominent ripple-band activity with spectral peaks from 150 to $200 \mathrm{~Hz}$ (Fig. 1C,D) that coincided with SWs in the stratum radiatum of CA1 (Fig. 1D). The DG-GCL LFP showed slow oscillations and large amplitude waves resulting in prominent $\delta$-band $(1-4 \mathrm{~Hz})$ activity in the spectrogram (Fig. 1B). Large amplitude waves in the DG can be induced by medial and lateral EC and CA3 input (Bragin et al., 1995; Penttonen et al., 1997). EC and CA3 afferents terminate, directly or indirectly, at different sites along the DG granule cell dendritic arbor: CA3 afferents terminate at the IML via mossy cells in the hilus of the DG, EC afferents terminate at the MML and OML (Li et al., 1994; Amaral et al., 2007; Scharfman, 2007). The 100- $\mu \mathrm{m}$ spacing of the electrode sites limited our ability to distinguish between all three layers of the ML. Therefore, we distinguished between IML and OML. By analyzing the CSD depth profile of the DG-GCL and ML channels (Fig. $1 D-G$ ), we were able to link large amplitude waves in our recordings to previously described EC or CA3 input to the DG. We identified DS type 2 (DS2; Fig. 1E; Bragin et al., 1995), DS type 1 (data not shown; Bragin et al., 1995b), and events with a DG-OML source (Fig. $1 F$ ). As the latter were previously 
A

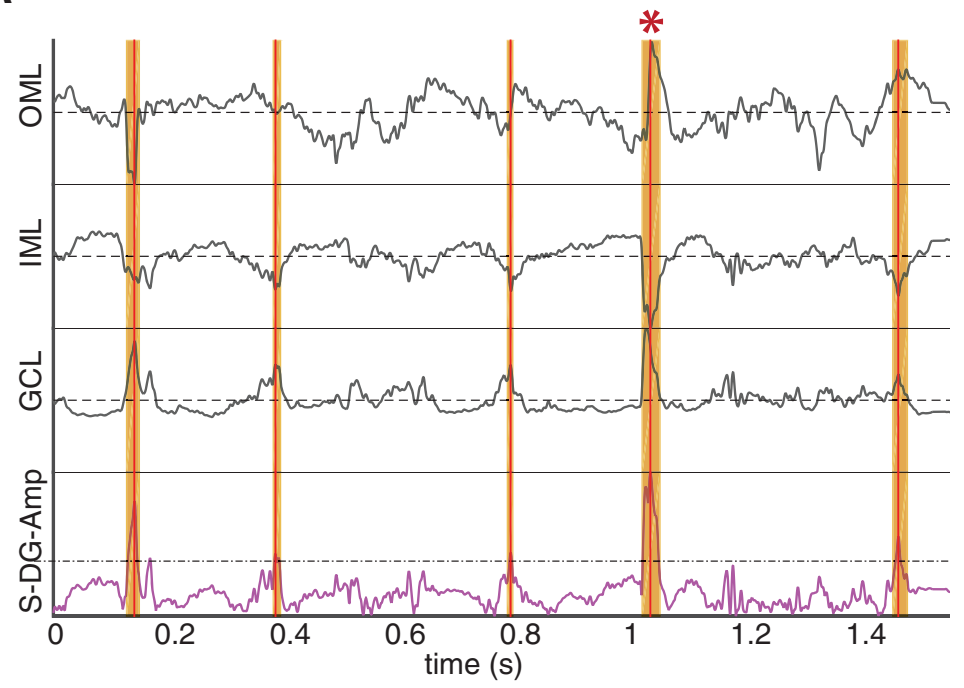

B

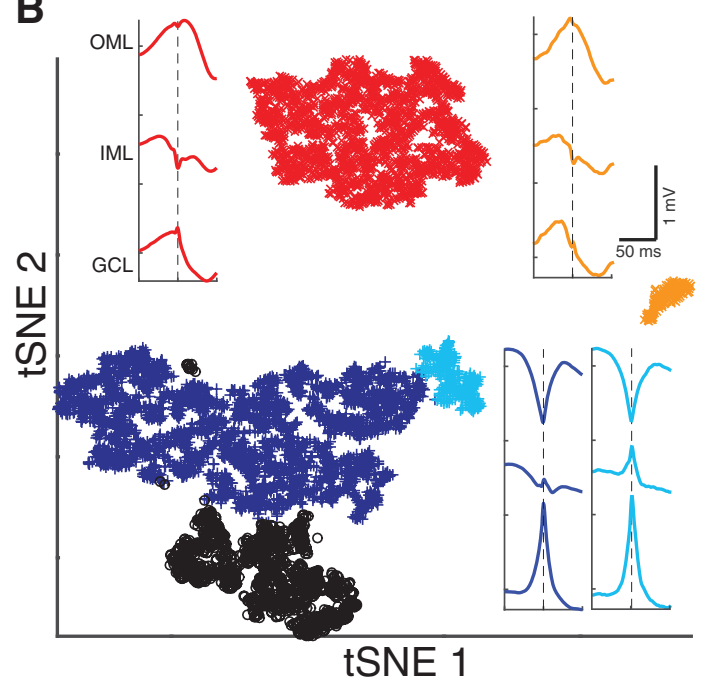

C
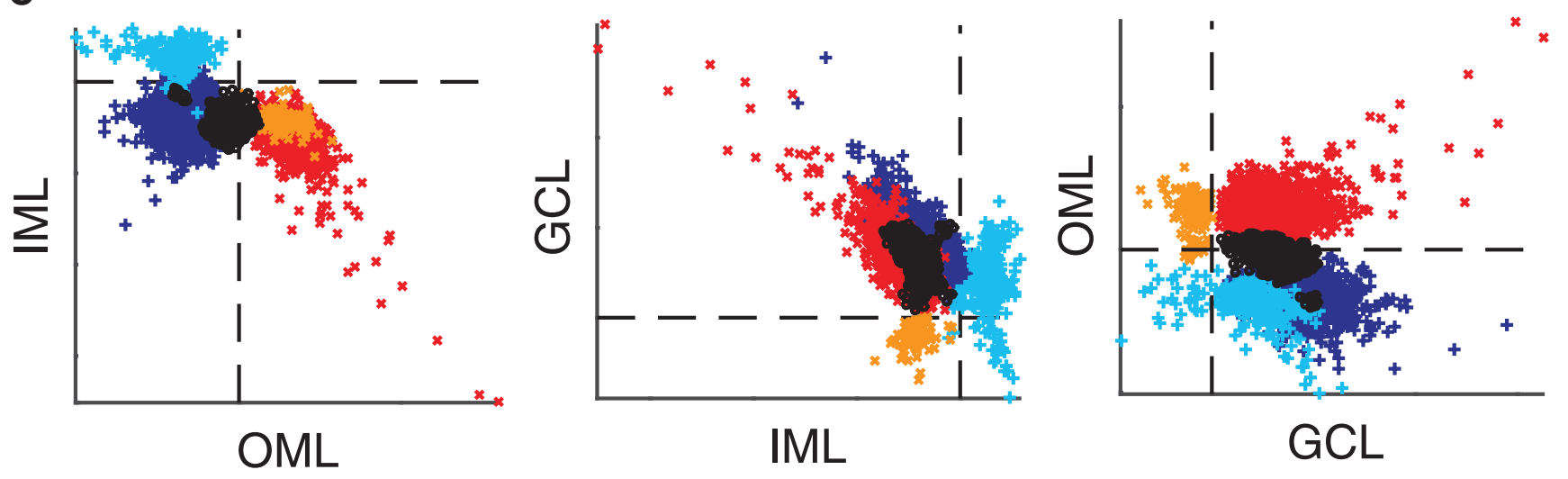

Figure 2. Dentate wave profiles cluster into distinct event types. $\boldsymbol{A}$, Example of dentate wave detection and the extraction of the waveform peak prominence. The gray traces show bandpass-filtered CSD (1-150 Hz) from the DG-0ML, DG-IML, and DG-GCL, and the dashed lines indicate the zero line. The magenta (bottom) trace shows the sum of the z-scored amplitude (S-DGAmp) of the traces above and the dot-dashed line indicates the threshold for event detection. Red lines show peaks exceeding three SD of the summed DG amplitude. Orange-shaded areas around red lines show periods for PPP determination. The red star indicates an example of amplitude maxima with a slight temporal offset. $\boldsymbol{B}$, An example t-SNE representation of the PPP from one sleep recording. Colors indicate individual PPP clusters determined by the DBSCAN algorithm, while black dots indicate unidentifiable clusters. Inset is the averaged DG LFP for the similarly colored clusters ( $\pm 50 \mathrm{~ms}$ centered on the peak detected in S-DG-Amp; channel identities are given in the top-left inset). $C$, CSD prominence of the DG channels plotted against each other, with clusters indicated as in $\boldsymbol{B}$. Examples in panels $\boldsymbol{A}-\boldsymbol{C}$ are from the same mouse.

undescribed, we named them DSWs. DSWs occurred independently or together with SWRs (Fig. $1 G$ ). DS2 events were characterized by a DG-GCL current source, and by DG-IML and DG-OML current sinks (Fig. $1 E$ ). DSWs were characterized by a DG-IML current sink, and by DG-GCL and DG-OML current sources (Fig. 1F,G).

\section{Dentate wave CSD profiles cluster into distinct event types}

The aim of this study was to identify experience-modulated LFP patterns in the DG. DG LFP events other than DSs may potentially reflect CA3-driven memory consolidation processes during sleep, much as CA1 SWRs do. Therefore, it was important to develop a method for detecting and classifying DG events on the DG CSD depth profile, independent of LFP activity present on non-DG hippocampal recording sites. In this way, we were able to relate the temporal and functional relationships of the identified DG events to CA3-CA1 activity patterns known to be relevant for memory consolidation during sleep. We identified clusters in the t-SNE representation of only the DG channels' CSD prominence profiles that represented distinct types of dentate activity (Fig. 2). Dentate waves were detected by thresholding the sum of the rectified z-scored DG amplitudes (S-DGAmp) of CSD channels in the dorsal blade of the DG (Fig. 2A) at three SD. Time windows of variable duration in which the $\mathrm{S}$ DG-Amp exceeded 1.5 SD of the mean were defined around the threshold-crossing maxima. The prominence of local maxima or minima in these time windows around the maximum SDG-Amp was used for clustering. We refer to this as PPP. Dimensionality reduction on the PPP was performed via t-SNE. Clusters within the two-dimensional t-SNE map were identified using the DBSCAN algorithm (see Materials and Methods). The cluster occupancy value in the following section is defined as the fraction of all clustered events that were assigned to a specific cluster. Two PPP clusters were present in all recordings analyzed ( $n=20$ from 7 animals), with: (1) current sinks in the IML and OML, and a current source in the GCL (cluster occupancy $58 \pm 18 \%$; Fig. $2 B, C$, blue cluster), or (2) a current sink in the IML and current sources in the OML and GCL (cluster occupancy $25 \pm 13 \%$; Fig. $2 B, C$, red cluster). Two other clusters were found in most of the recordings: (3) a current sink in the OML and current sources in the IML and GCL, which were found in 
A

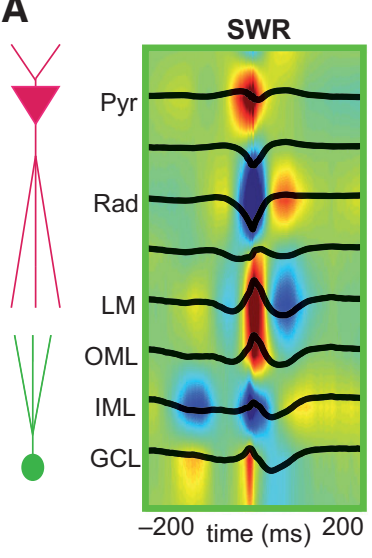

B

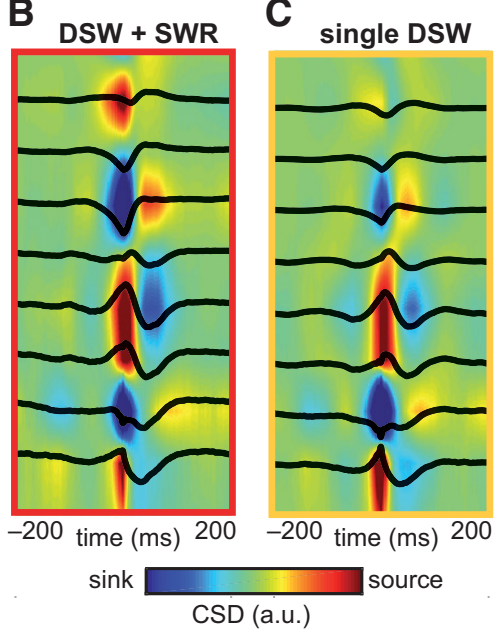

D

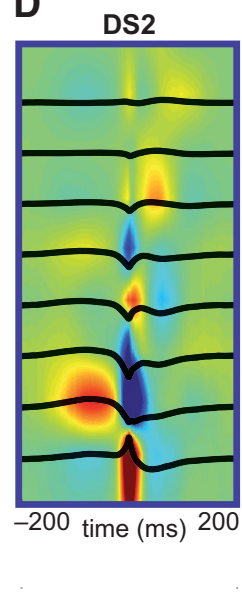

E

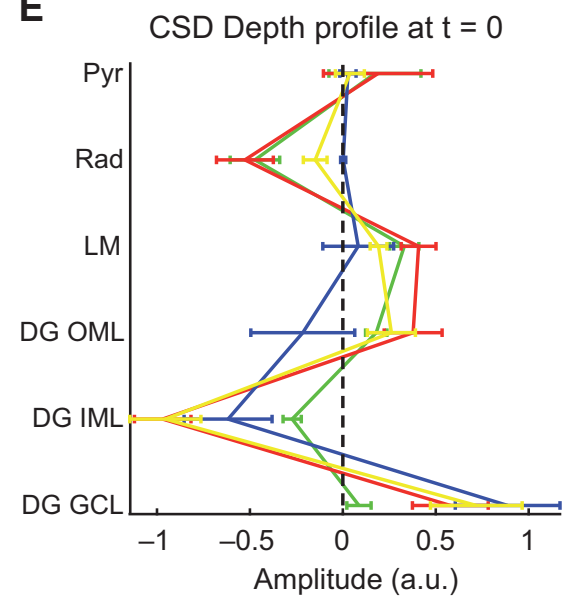

$\mathbf{F}$

Occurrence of DSW or DS2 G relative to SWR
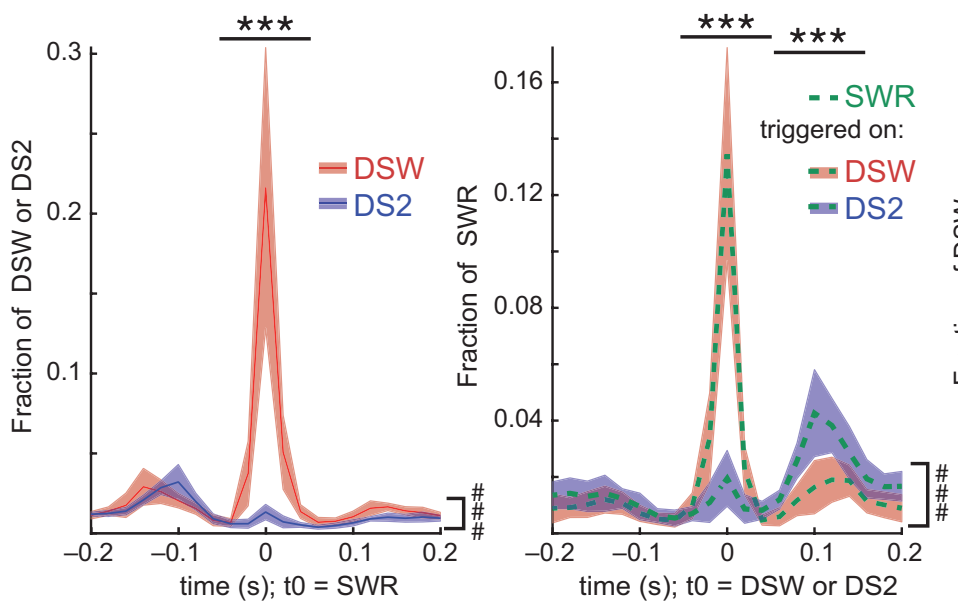

Occurrence of SWR relative to DSW or DS2

H
Occurrence of DSW relative to DS2

\section{Occurrence rate during SWS}
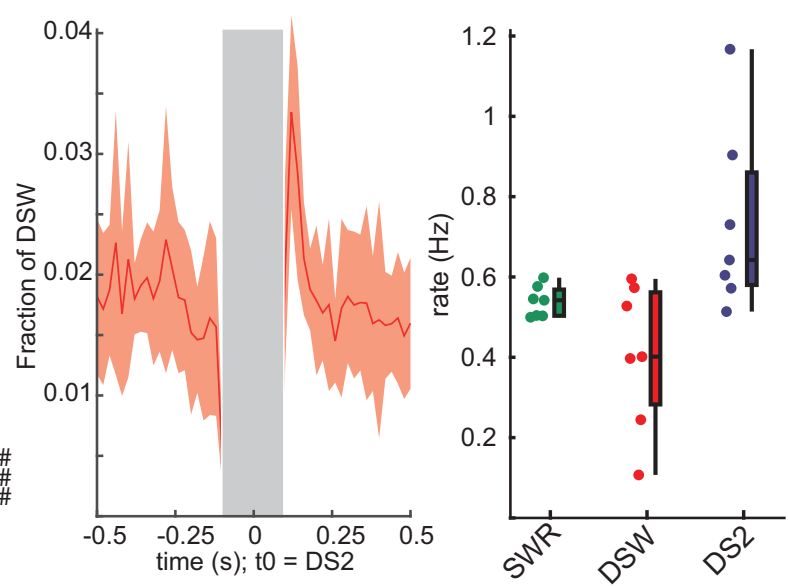

Figure 3. Unique relationship of novel DSW cluster to CA1 SWR. A-D, Triggered average depth profiles of one example baseline sleep recording. Traces indicate LFP, colored background indicates CSD. CSD color axis ranges from -3 to +3 SD of the mean of the respective triggered averages. On the left, the location of CA1 pyramidal (magenta) and DG granule cells (green) are schematically shown. Visualized network patterns are: CA1 SWR $(\boldsymbol{A})$, DSW + SWR $(\boldsymbol{B})$, defined by DSW peak that occurs within $\pm 50 \mathrm{~ms}$ to CA1 SWR SW trough, single DSW (C), i.e., all DSWs that do not match the criteria of DSW + SWR (compare to $\boldsymbol{B}$ ), and DS2 (D). Please note the similarity between CA1 SWR and DSW depth profiles. $\boldsymbol{E}$, Triggered average CSD depth profiles of CA1 SWR (green), DSW + SWR (red), DSW (yellow), and DS2 (blue) for all baseline sleep recordings analyzed ( $n=7$ mice) at the peak/t0 (compare $\boldsymbol{A}-\boldsymbol{D}$ ). Error bars show SD. F, G, Temporal coupling of CA1 SWRs with DSWs or DS2s. Shaded regions cover SD $(n=7)$. $\boldsymbol{F}, 0$ ccurrence of DSW (red) or DS2 (blue) in temporal relationship to SWR. The CCG shows the fraction of DSWs or DS2s that occurs in a distinct temporal relationship to SWR ${ }^{* * *} p<0.001$ comparison between the occurrence of DSWs and DS2s from -0.05 to $+0.05 \mathrm{~s}$, calculated with the Tukey's HSD post hoc test after a significant effect of the interaction between type of co-occurring event (DSW or DS2) and temporal relationship (from -0.25 to $+0.25 \mathrm{~s}$, using time bins of $0.1 \mathrm{~s}$ duration, centered on $-0.2,-0.1,0,0.1$, and $0.2 \mathrm{~s}$ ) with a two-way ANOVA for repeated measures $\left(F_{(4,24)}=38.05\right.$; \#\#\#p $\left.<0.001\right)$. G, Occurrence of SWR dependent on DSW (red) or DS2 (blue), shown by $C C G(* * * p<0.001$ comparison between the occurrence of SWRs in relation to DSWs and SWRs in relation to DS2s from -0.05 to $+0.05 \mathrm{~s}$ and from +0.05 to $+0.15 \mathrm{~s}$ calculated as in $F$; $F_{(4,24)}=46.74 ; \# \#$; 0.001$)$. H, CCG of DSWs and DS2s. Red-shaded regions cover the SD $(n=7)$, the gray-shaded region marks the minimum interevent interval of $100 \mathrm{~ms}$ around the DS2 peak. Note the difference in timescale in $\boldsymbol{F}, \boldsymbol{G}$. I, Event rate of SWR (green), DSW (red), and DS2 (blue) during the SWS phases of baseline recordings for $n=7$ mice. Boxplots indicate median and interquartile range; whiskers extend to the furthest outliers.

$85 \%$ of the analyzed recordings (cluster occupancy $10 \pm 7 \%$; Fig. $2 B, C$, cyan cluster), and (4) current sinks in the IML and GCL and a current source in the OML, which were detected in $75 \%$ of the analyzed recordings (cluster occupancy $2 \pm 1 \%$; Fig. $2 B, C$, orange cluster). In $45 \%$ of the recordings, we found additional clusters that could not be assigned to any of the meta clusters 1-4 (cluster occupancy $13 \pm 15 \%$; Fig. 2B,C, black cluster). We therefore restricted our subsequent analysis to clusters 1 and 2 because they were present in all recordings. The overall contribution of clusters 3 and 4 was small or variable.

\section{Unique relationship of novel DSW cluster to CA1 SWRs}

To relate our clusters to previously described dentate LFP patterns (i.e., DS2), we compared instantaneous CSD depth profiles and the temporal relationship of the clusters to CA1-SWRs (Fig. $3 A$ ) during baseline sleep in the home cage.

We use the term DSW to refer to the novel CSD pattern in the DG detectable by our cluster analysis (cluster 2, red cluster in Fig. $2 B, C$ ) in every recording because the triggered average CSD (Fig. 3B,C) showed a similar distribution of current sources and current sinks as the average CSD of SWRs (Fig. $3 A, E$ ). Like CA1SWRs, DSWs showed a DG-IML sink. Furthermore, the DSW cluster co-occurred with a sink in CA1 stratum radiatum similar to CA1-SWR depth profiles (Fig. 3C). DSWs and CA1-SWRs frequently co-occurred, that is, $32.46 \pm 12.66 \%$ of DSWs were detected within $\pm 50 \mathrm{~ms}$ of the SW trough of a CA1-SWR (Fig. $3 F)$. We will refer to this as a DSW-SWR pair. A smaller fraction of all SWRs $(20.5 \pm 6.0 \%)$ co-occurred with a DSW in the same 

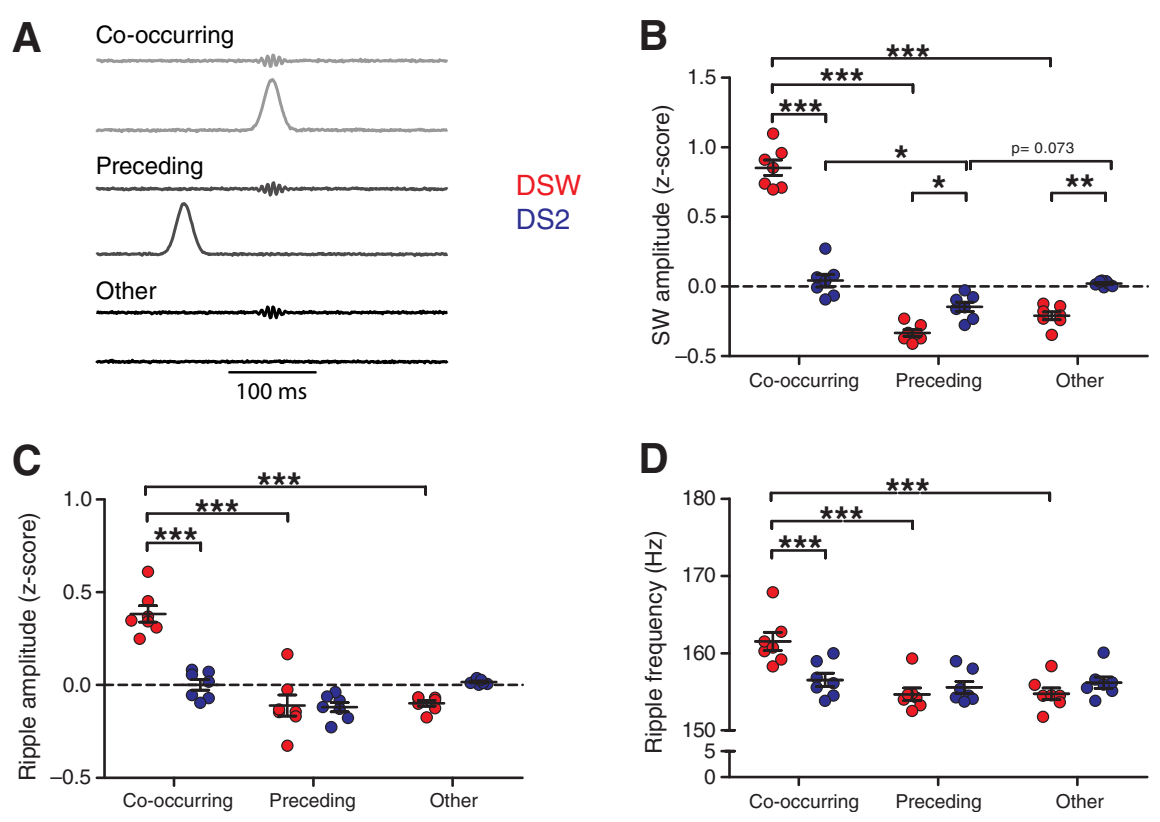

Figure 4. DSW-SWR pairs modulate CA1 SW and ripple properties $A$, Examples of temporal sequence or co-occurrence patterns of SWR (upper traces) and DSW or DS2 (lower traces), as shown in Figure 3C. SWR and DSW/DS2 events are defined as co-occurring when they appear within a time window of $\pm 50 \mathrm{~ms}$. Preceding DSW/DS2 precede SWRs by $50-150 \mathrm{~ms}$. Events with any other temporal relationship were labeled as 0ther. $\boldsymbol{B}-\boldsymbol{D}$, Sharp-wave amplitude $(\boldsymbol{B})$, ripple amplitude $(\boldsymbol{C})$, and ripple frequency $(\boldsymbol{D})$, dependent on temporal relationship to DSW (red) or DS2 (blue). Each dot shows one animal $(n=7) ;{ }^{*} p<0.05,{ }^{*} p<0.01$, ${ }^{* * *} p<0.001$, calculated with the Tukey's HSD post hoc test, after a significant effect of the interaction between type of co-occurring event (DSW or DS2) and temporal relationship (c0-occurring, preceding, or other) calculated with a two-way ANOVA for repeated measurements $\left(F_{(2,12)}=122.7 ; p<10^{-7}\right)$.

time window (Fig. 3G). The CSD depth profile of DSW-SWR pairs at $t=0$ did not show differences on the DG channels compared with single DSWs, but only on the CA1 channels (Fig. 3E, red vs. yellow). Single DSWs also exhibited small current sinks in the CA1 stratum radiatum, resembling those seen during CA1 SWRs. However, these DSWs did not co-occur with a thresholdcrossing ripple (Fig. $3 C$ ). We also observed current sources in CA1 stratum lacunosum moleculare during single DSWs (Fig. $3 C$ ), which resembled the return currents of the CA1 SW. Both CA1 stratum radiatum sinks and lacunosum source potentials in the CSD had lower amplitudes during single DSWs than during DSW-SWR pairs (Fig. 3E).

The other cluster observed in all recordings was composed of DS2s (cluster 1, blue cluster in Fig. $2 B, C$ ). DS2 events have current sinks in DG-OML and DG-IML and a current source in the DG-GCL (Fig. 3D). During DS2 events, we did not find a significant co-activation/current sink in CA1 (Fig. $3 E$ ). DS2 events preferentially occurred $50-150 \mathrm{~ms}$ before a SWR, with $11.0 \pm$ $2.0 \%$ of DS2 events preceding CA1-SWRs (Fig. 3F) and $14.7 \pm 3.8 \%$ of SWRs following a DS2 (Fig. 3G). DS2s tended to precede DSWs by $100-150 \mathrm{~ms}$ (the minimal interevent interval for the detection of DSWs and DS2s was $100 \mathrm{~ms}$; Fig. $3 \mathrm{H}$ ). DSW rates were generally the lowest and DS2 rates the highest (Fig. 3I).

\section{DSW-SWR pairs modulate SWR properties}

The temporal relationship between SWRs and DSWs suggested a functional connection between the two events. As it is known that DS2 events inhibit SWR generation in rat hippocampus in vivo (Bragin et al., 1995), we investigated the modulation of functional properties of SWRs conditioned on their temporal relationship to DSWs or DS2s. Given the unique temporal relationships that we found for DSWs and DS2s to SWRs (compare Fig. $3 F, G)$, we tested the ripple properties for three groups (Fig. 4A): (1) the co-occurring group comprised SWRs that occurred within a time window of $\pm 50 \mathrm{~ms}$ to a DSW or DS2, (2) the preceding group comprised SWRs that were preceded by a DSW or DS2 within 50-150 ms, and (3) the other group comprised any temporal relationship of SWRs to a DSW, or to a DS2, that is not covered by the cooccurring or the preceding groups.

We found that stratum radiatum SW amplitudes were significantly higher when they co-occurred with DSWs within $\pm 50 \mathrm{~ms}$ (Fig. $4 B$ ). Furthermore, ripple amplitudes and mean ripple frequencies were increased when the SWR co-occurred with DSWs within $\pm 50 \mathrm{~ms}$ (Fig. 4C,D).

SW amplitudes of SWR events that were preceded by DS2 within $50-150 \mathrm{~ms}$ were significantly lower in amplitude than SWs co-occurring with DS2 and tended to be smaller than SWs in the other group (Fig. $4 B$ ). Ripple amplitudes and frequencies, however, were not dependent on the temporal relationships to DS2 events (Fig. 4C,D). The lack of effect on ripple amplitude (see Headley et al., 2017) in our analysis may result from our inclusive detection with low thresholds and wide bandpass filter.

Taken together, large amplitude and fast SWR tended to cooccur with DSW, whereas DS2 events were more likely to precede SWRs of varied strengths. This finding indicates that the CSD patterns we detected were consistently related to fast oscillatory activity in CA1 across different animals, confirming that our dentate-wave classification led to the identification of functionally relevant and distinct physiological events. The temporal and functional correlation of DSWs and SWRs suggested a role for DSWs in memory consolidation.

\section{DSWs and DS2s mediate distinct patterns of multiunit activity (MUA)}

As previous studies in rats indicated that different patterns of spiking activity along the CA1-DG axis accompany CA1 SWR and DS2 events (Bragin et al., 1995; Penttonen et al., 1997), we next analyzed the temporal relationship between CA1p and DGGCL MUA and SWR, DSW, or DS2. We found that MUA in CA1p differentially correlated to DSW or DS2 events. Previous work showed a co-activation of CA1p and DG GCs during SWRs in urethane-anesthetized rats (Penttonen et al., 1997). We observed an increase of MUA in CA1p and DG-GCL 100 ms before DSW or SWR events (Fig. 5A,B). MUA rapidly decreased after the occurrence of DSW or SWR peaks. The simultaneous MUA increase and decrease in CA1p and DG-GCL indicate that MUA in both regions may be driven by the same source during DSWs.

Previous studies on DS2s recorded from rats described an increase in DG-GCL MUA during DS2s, but a decrease in CA1p MUA and SWR rates (Bragin et al., 1995; Penttonen et al., 1997). 
A
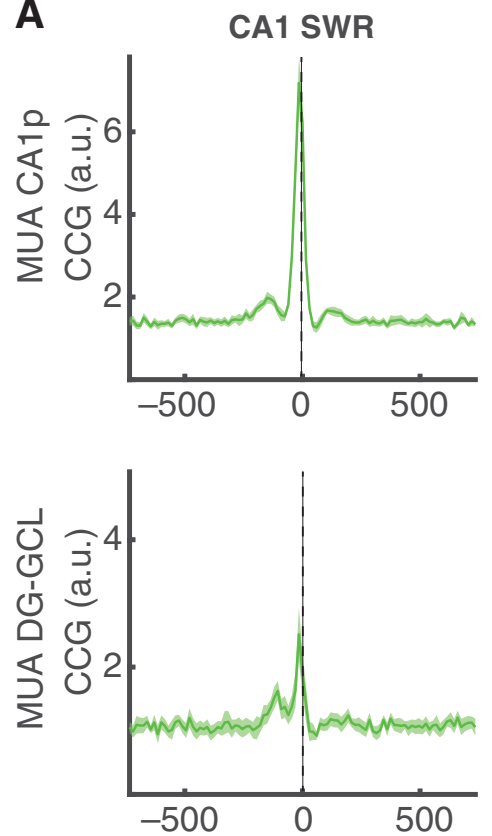

D
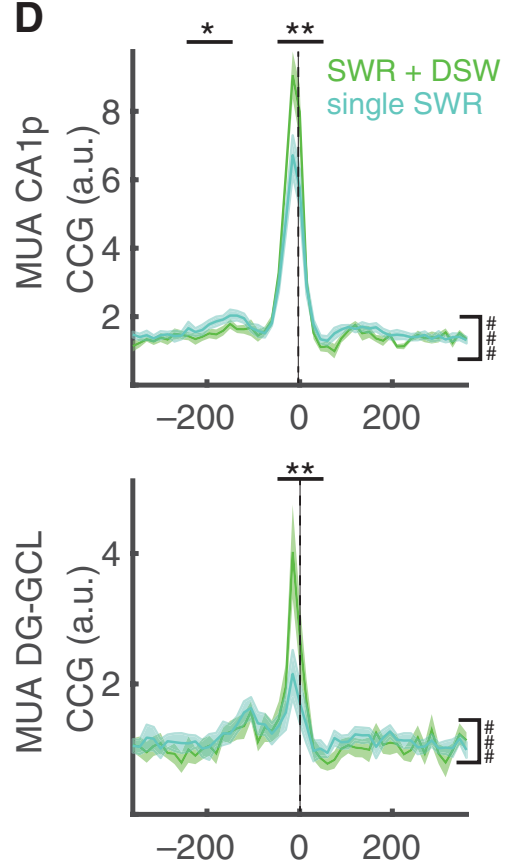

time (ms); t0 = CA1 SWR
B
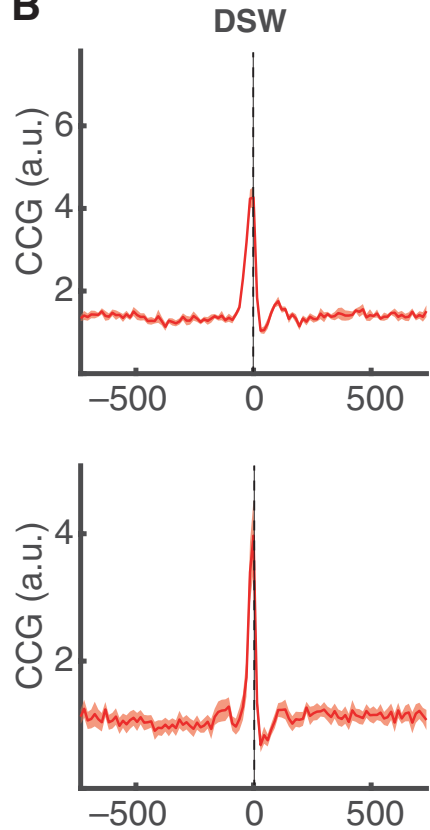

E
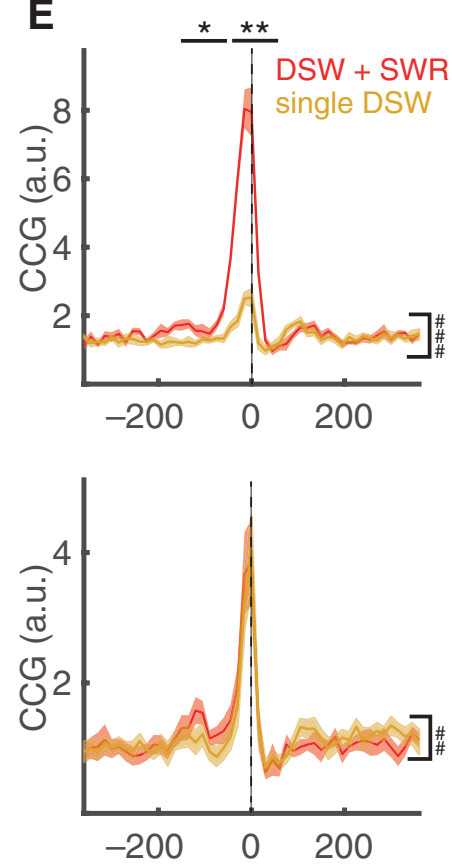

time (ms); t0 = DSW
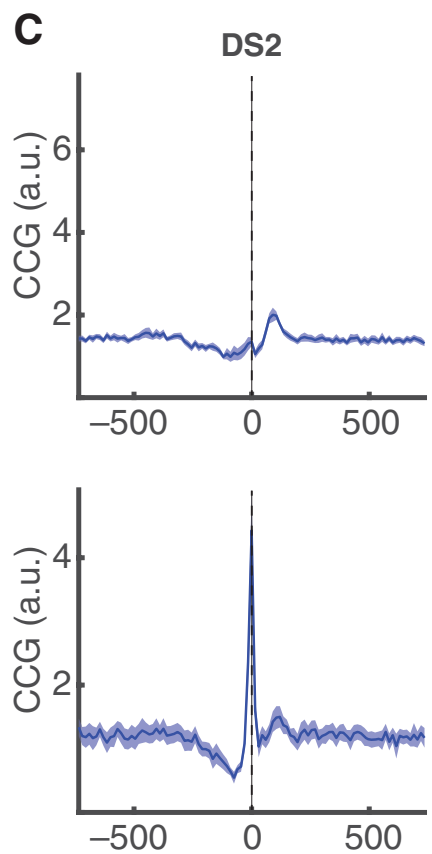

F
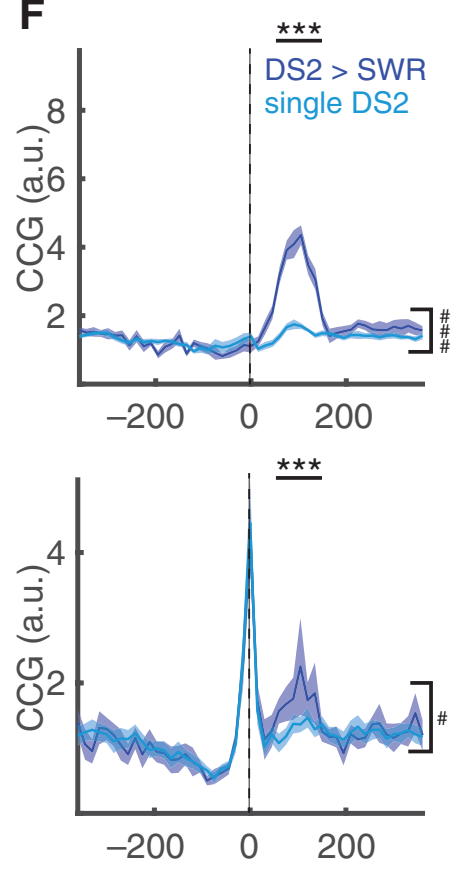

time (ms); t0 = DS2

Figure 5. DSWs and DS2s mediate distinct patterns of MUA. $\boldsymbol{A}-\boldsymbol{C}$, MUA in temporal relationship to SWR (A), DSW (B), and DS2 (C) in CA1p (top panel) and DG-GCL (bottom panel). Error bars indicate SEM $(n=7)$. The CCG is scaled by the product of firing rates to asymptote to $\sim 1$. D-F, MUA in CA1p (top) or DG (bottom) dependent on the co-occurrence of SWRs with DSWs within $\pm 50 \mathrm{~ms}(\boldsymbol{D})$, the co-occurrence of DSWs with SWRs within $\pm 50 \mathrm{~ms}(\boldsymbol{E})$, or the temporal sequence of DS2s followed by SWRs within 50-150 ms $(\boldsymbol{F})$. Comparisons between MUA during SWR + DSW and single SWR (D), DSW + SWR and single DSW $(\boldsymbol{E})$, and between DS2+SWR and single DS2 $(\boldsymbol{F})$ were calculated with the Tukey's HSD post hoc test $\left(^{*}\right.$ indicates significance levels) following a significant effect of interaction (indicated by \#) between type of co-occurring event (single event or event combination) and temporal relationship ( $\pm 700 \mathrm{~ms}$ of which $\pm 300 \mathrm{~ms}$ are shown in $\boldsymbol{D}-\boldsymbol{F}$ to highlight the interval with significant differences) calculated with a two-way ANOVA for repeated measurements using 100-ms time bins centered around $t=0$ [CA1p/DG MUA: (D) $\left.F_{(14,84)}=13.28 / F_{(14,84)}=6.72 ;(\boldsymbol{E}) F_{(14,84)}=47.85 / F_{(14,84)}=2.38 ;(\boldsymbol{F}) F_{(14,84)}=32.25 / F_{(14,84)}=2.23\right]$. Error bars indicate SEM $\left(n=7\right.$ mice; ${ }^{*} / \# p<0.05, * * / \# \# p<0.01$, ****\#\#\# <0.001).

In our dataset, MUA was suppressed $\sim 100 \mathrm{~ms}$ before the appearance of DS2 events in the DG-GCL and CA1p but increased again in DG GCL around the amplitude peak of DS2 (Fig. $5 C$ ). The MUA increased in CA1p 50-150 ms after the DS2 peak (Fig. $5 C$ ). This pattern suggests a sequential activation of the DG and CA1 during or after DS2 events. Similar activation patterns also accompany cortical down-to-up state transitions (Isomura et al., 2006; Sullivan et al., 2011).

As expected, MUA in CAlp increased during SWRs (Fig. $5 A$ ). Similarly, MUA in DG-GCL concomitantly increased with either DSWs or DS2 (Fig. 5B,C). When SWRs co-occurred with DSWs, MUA in CA1p and DG were significantly higher than 

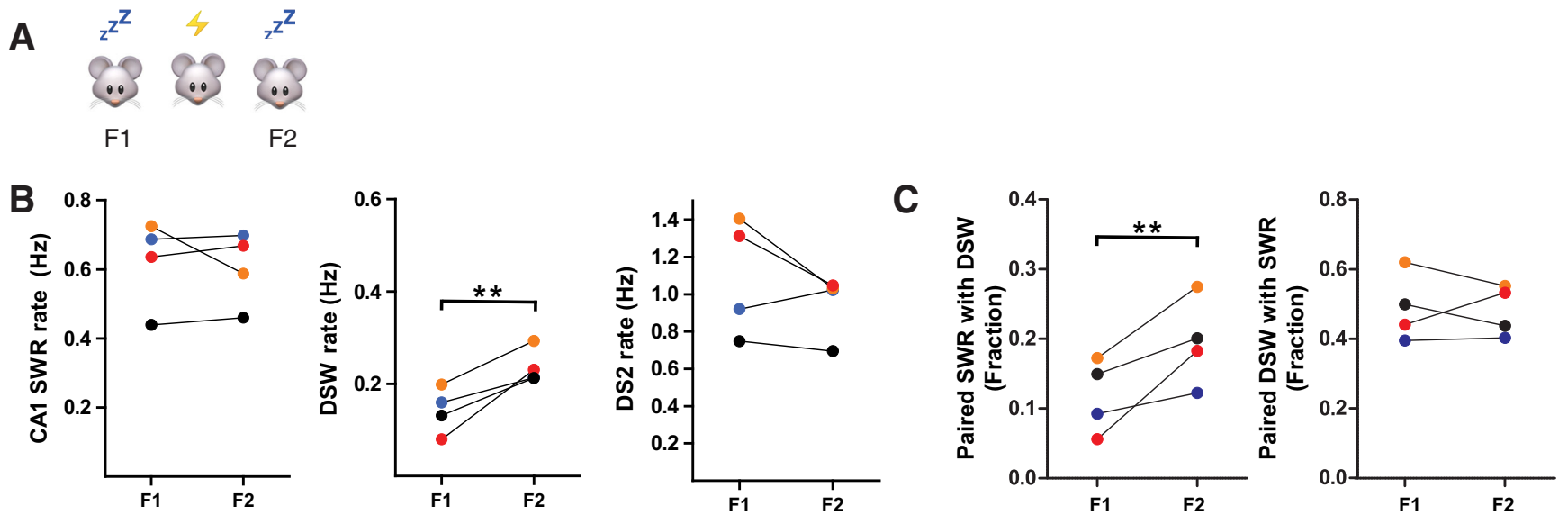

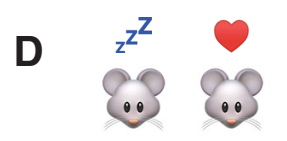

R1

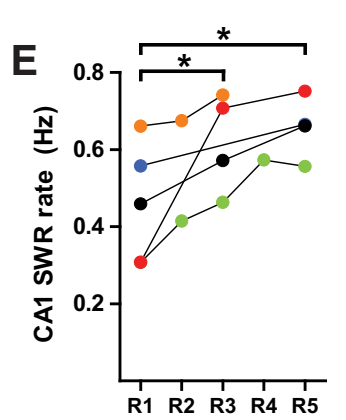

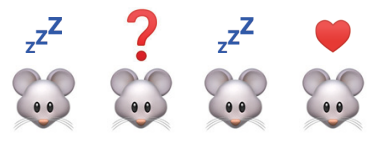

R3

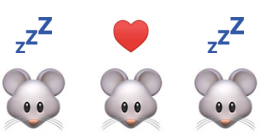

R4

R5
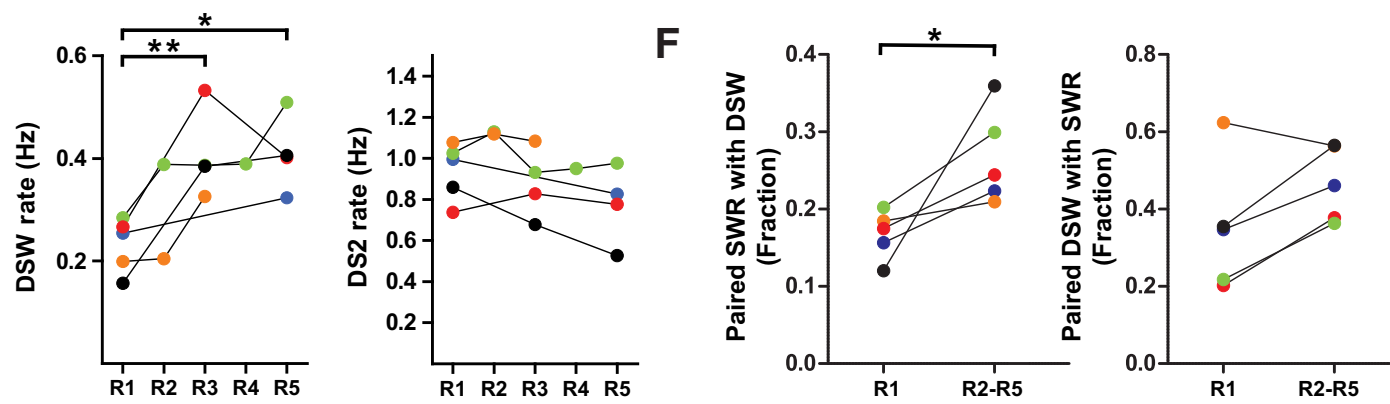

Figure 6. DSW rate during initial SWS was increased by previous learning experiences. $A$, Experimental design of the cued fear conditioning. Sleep sessions before (F1) and after one fear conditioning session (F2) were recorded. $\boldsymbol{B}$, Change in occurrence rates of CA1 SWR (left panel), DSW (middle panel), DS2 (right panel) in the first 10 min of sleep after fear conditioning $(n=4)$. C, Fraction of SWR co-occurring with DSWs (left) and DSWs co-occurring with SWRs (right) within $\pm 50 \mathrm{~ms}(n=4)$. $D$, Experimental design of spatial learning trial. After a sleep session (R1), male mice were exposed to female mice in a beaker of a square arena. The second session (i.e., between R2 and R3) was performed without female mice, and the following two sessions were performed with females present in the beaker. Sleep was recorded before, between, and after behavioral sessions (R1-R5). $\boldsymbol{E}$, Change in occurrence rates of CA1 SWR (left panel), DSW (middle panel), DS2 (right panel) in the first 10 min of sleep after spatial learning $(n=5)$. $\boldsymbol{F}$, Fraction of SWRs co-occurring with DSWs (left) and DSWs co-occurring with SWRs (right panel) within \pm 50 ms before and after spatial learning. The post-training datapoint R2-R5 shows the mean value of the sleep sessions R2-R5 obtained from the respective animal ( $n=5)$. Note, colors in $\boldsymbol{B}, \boldsymbol{C}, \boldsymbol{E}, \boldsymbol{F}$ are matching individual mice. Tukey's HSD post hoc test $\left({ }^{*} p<0.05,{ }^{* *} p<0.01\right)$ after significant effect of the interaction between type of event (CA1-SWR, DSW, and DS2) and sleep phase (F1 and F2 in $\boldsymbol{B}$, and R1 and R3 or R5 in $\boldsymbol{E})$ calculated with a two-way ANOVA for repeated measurements $\left(F_{(2,6)}=14.80, p<0.01\right.$ in $\boldsymbol{B} ; F_{(2,6)}=16.64, p<0.01$ in $\boldsymbol{E}$ with R1 vs R3; $F_{(2,6)}=11.19, p<0.01$ in $\boldsymbol{E}$ with R1 vs R5). We did not perform statistical tests on the trials R2 and R4 because of insufficient sleep epochs during these sessions. For statistical analysis of $\boldsymbol{C}, \boldsymbol{F}$, we normalized the postexperience datapoints F2 or R2-R5 to the preexperience datapoint F1 or R1, respectively, and performed an analysis using the Tukey's HSD post hoc test after a significant effect of the interaction between event pairs or sequences (SWRs paired with DSWs within $\pm 50 \mathrm{~ms}$, DSWs paired with SWRs within $\pm 50 \mathrm{~ms}$, DS2s followed by SWRs within 50 150 ms, SWRs following DS2s within 50-150 ms; data of sequential DS2 and SWR occurrence are not shown) and sleep phase (F1 and F2 in $\boldsymbol{B}$, and R1 and R2-R5 in $\boldsymbol{E}$ ) calculated with a twoway ANOVA for repeated measurements $\left(F_{(3,9)}=16,36, p<0.001\right.$ in $\boldsymbol{C} ; F_{(3,12)}=4.24, p<0.05$ in $\left.\boldsymbol{F}\right)$.

MUA during single SWRs (Fig. 5D). This result matches our finding that SWRs co-occurring with DSWs have higher ripple frequencies and amplitudes, and higher SW amplitudes than single SWR (Fig. $4 B-D$ ). In contrast, when DSWs co-occurred with SWRs, MUA was increased in CAlp but in DG remained comparable to the single DSW case (Fig. $5 E$ ). When SWRs followed DS2 events, MUA in CA1p peaked $\sim 100 \mathrm{~ms}$ after the DS2 maximum (Fig. 5F). Likewise, MUA in DG increased 50-150 ms after DS2, suggesting that some of the SWRs that followed a DS2 co-occurred with DSWs. DSWs and DS2s exhibited distinct temporal trajectories of MUA patterns. During DSWs, a slow, concomitant increase of MUA in CA1p and DG-GCL was observed, which was immediately inhibited after the DSW peak. In contrast, MUA was suppressed in CA1p and DG-GCL just before DS2 events but was increased sequentially, that is, first in DGGCL and then in CAlp. These results suggest that DSWs and
DS2s are LFP correlates of distinctly modulated patterns of excitation and inhibition in hippocampal subregions.

\section{DSW rate is robustly increased by previous learning experiences}

DSWs showed a distinct temporal relationship to SWRs and their properties. SWRs are crucial for memory consolidation, and the SWR occurrence rate in rats is increased after learning (Eschenko et al., 2008; Girardeau et al., 2014). We, therefore, examined whether the DSW or DS2 occurrence rate was also modulated by a learning experience. To this end, we conducted hippocampus-dependent fear conditioning and spatial learning experiments and recorded sleep epochs immediately before and after behavioral testing (Fig. 6). Fear conditioning was performed with a conditioning protocol containing six CS-US pairings. Spatial learning was assessed via an ecologically relevant stress- 


\section{Cued fear conditioning}

A
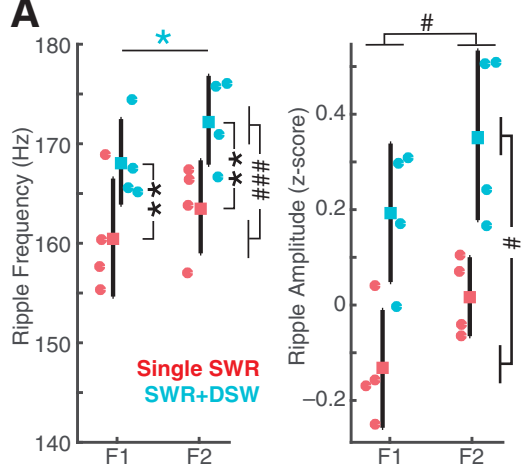

C
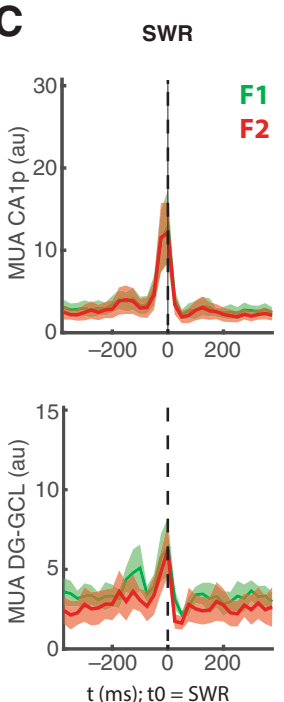

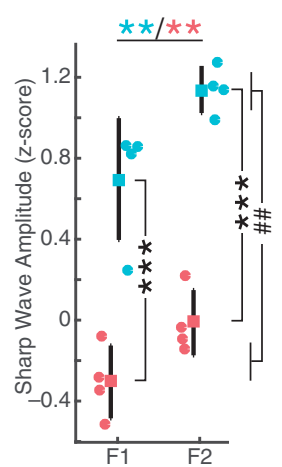

DSW
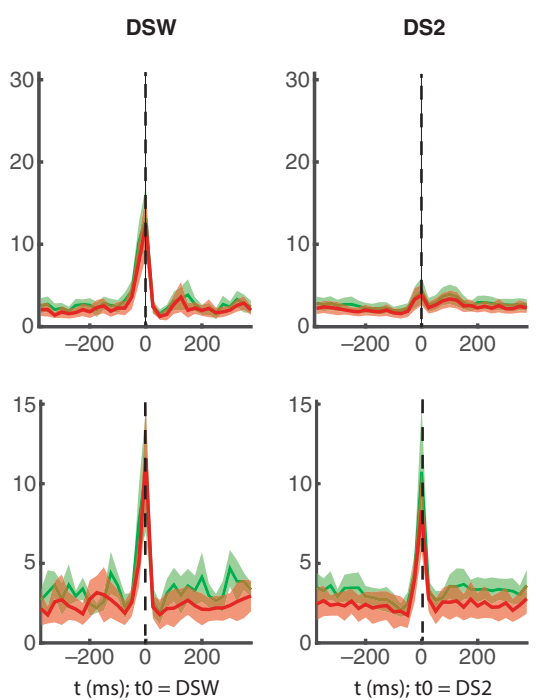

Spatial learning

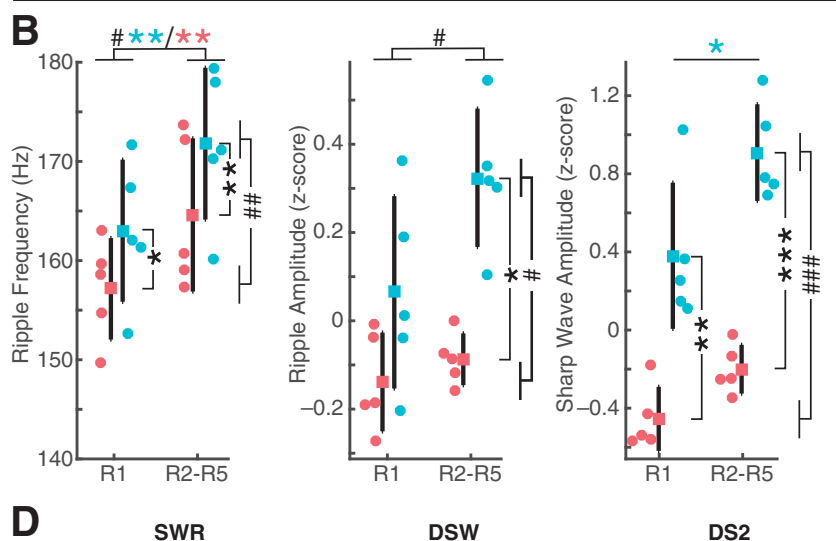

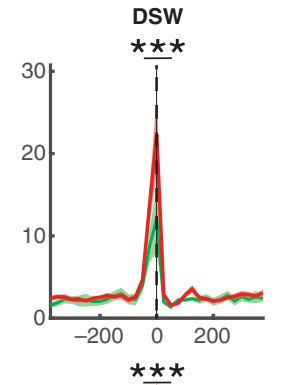
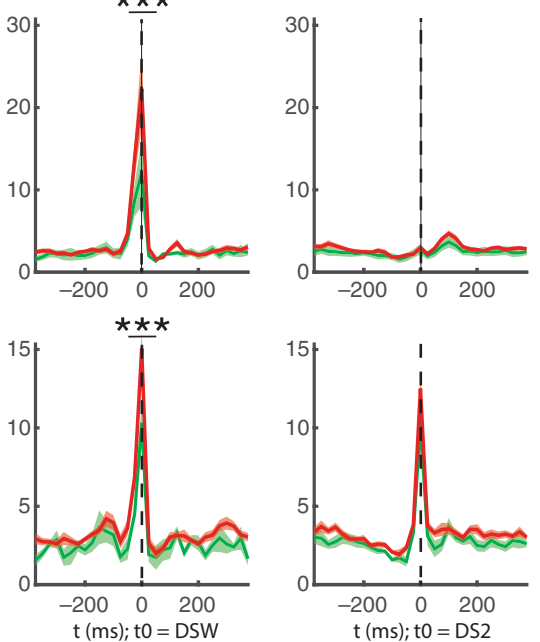

Figure 7. Experience-dependent change in SWR and MUA during NREM sleep. All panels show data analyzed during the first $10 \mathrm{~min}$ of NREM sleep before and after the learning trials of the fear conditioning $(\boldsymbol{A}, \boldsymbol{C})$ and spatial learning tests $(\boldsymbol{B}, \boldsymbol{D})$. For the spatial learning test, the mean of the available sleep phases R2-R5 is shown. $\boldsymbol{A}, \boldsymbol{B}$, SWR properties of single SWR (red) or SWR co-occurring within $\pm 50 \mathrm{~ms}$ of DSW (blue) before and after $(\boldsymbol{A})$ fear conditioning and $(\boldsymbol{B})$ spatial learning. Round dots show data points of individual mice. Squared dots show mean, bars show SD. C, D, CCGs of CA1p or DG-GCL MUA showing temporal relationship to SWRs (respective left panel), DSWs (respective middle panel), and DS2s (respective right panel) before (green) and after learning (red). Error bars indicate SEM. The colored asterisks indicate the comparisons between sleep phases F1 (before learning) and F2 (after learning; $A$ ) and between sleep phases R1 (before learning) and R2-R5 (after learning; $\boldsymbol{B}$ ) for the single SWR (red asterisk) or the paired SWR (blue asterisk). The black asterisks indicate the comparisons between single SWR and paired SWR at a specific time point, namely, F1 or F2 (A) and R1 or R2-R5 (B). In D, significance was observed for the \pm 50 -ms bin around SWRs or DSWs; however, for DG-GCL MUA around SWR the increased CCG synchrony was also significant in the -150- to - 50-ms bin. All comparisons were analyzed by Tukey's HSD post hoc tests after two-way ANOVA for repeated measures. For all colors: ${ }^{*} p<0.05,{ }^{* *} p<0.05,{ }^{* * *} p<0.001$. \# symbols indicate the effect of the factor sleep phase (horizontal lines), or of the factor "single or paired event" (vertical lines) as analyzed by two-way ANOVA for repeated measures; \#p <0.05, \#\#p <0.01, \#\#p <0.001.

free learning paradigm in which male mice were exposed to an arena with female mice (Fellini and Morellini, 2013). To test whether the training trials induced long-term memory consolidation of the newly learned experiences, we analyzed memory recall $1 \mathrm{~d}$ after training. For recall of the cued fear conditioning paradigm, mice were exposed to 20 CS presentations, each lasting $20 \mathrm{~s}$ (without US). All mice showed robust freezing behavior during the first five CSs (group mean time spent freezing $=42.30$ $\pm 3.74 \%$ ), indicating that they recalled the association between $\mathrm{CS}$ and US. Memory recall in the spatial learning paradigm was tested by analyzing whether mice had spent more time at the females' original position as compared with the other corners when placed in an arena with no female mice present. One-way ANOVA for repeated measurements (with box as within-group factor) indicated that mice spent more time at the females' box as compared with the other two boxes (time at females' box = $265.4 \pm 125.4 \mathrm{~s}$, time at the opposite box $=92.0 \pm 53.7 \mathrm{~s}$, time at adjacent box $=105.4 \pm 25.0 \mathrm{~s}$; effect of "box" $F_{(2,14)}=6.48$; $p<0.05$; Turkey's HSD post hoc comparison between time at the females' box and time at the other two boxes $p<0.05$ ). To test the hypothesis that activity in the DG correlates to memory consolidation processes within the cortico-hippocampal network, we analyzed the occurrence rates of SWR, DSW, and DS2. We classified DSWs and DS2s for all sleep sessions of a respective behavior trial day and animal in one cluster analysis. Interindividual and intraindividual variability in the sleep recordings, which resulted from differences in sleep onset and sleep architecture, made the quantification of learning-induced changes in spontaneous sleep-related LFP patterns challenging. We therefore focused on the first NREM periods, analyzing 10 min of NREM sleep immediately following sleep onset. During this initial NREM following exposure, DSWs occurred more frequently after fear conditioning (Fig. 6B) or spatial learning (Fig. 6E) as compared with sleep before testing. The occurrence of DS2s, in contrast, remained unchanged during the analyzed recording interval. SWR rates were selectively 
increased by spatial learning (Fig. $6 E$ ) but not by fear conditioning (Fig. 6B).

As we observed a temporal coupling between SWRs and DSWs or DS2s (Fig. $3 F-H$ ), we examined whether a previous experience in a learning task altered the fractions of co-occurring SWRs and DSWs, or the fraction of DS2s followed or preceded by a SWR within $50-150 \mathrm{~ms}$. We found a significant effect of the interaction between the type of event and the sleep phase (baseline sleep before the learning trials vs sleep after the learning trial, two-way repeated measures ANOVA: $F_{(3,9)}=7.89, p<0.01$ ). Post hoc analyses indicated that the fraction of SWRs co-occurring with DSWs was increased during the sleep phase following the learning trials of the fear conditioning (Fig. 6C, left) and spatial learning tests (Fig. $6 F$, left). In contrast, the learning experiences had no significant effect on either the fraction of DSWs that occur paired with SWRs (Fig. 6C,F, right) or on the fraction of SWRs or DS2s occurring in sequence with each other (data not shown).

\section{Learning experiences modulate SWR properties and MUA related to DSWs}

As the properties of SWRs (Fig. 4) and MUA during SWRs in CAlp and DG (Fig. 5) depended on the temporal relationship between SWRs and DSWs, we tested whether experience in a learning trial may not only increase the fraction of SWRs cooccurring with DSWs (as shown in Fig. 6C,F) but also alter the properties of SWRs or SWR-triggered, DSW-triggered, or DS2triggered MUAs in CA1p or DG.

In line with our previous observation that DSWs modulated SWR properties (Fig. 4), SWRs paired with DSWs had higher ripple frequencies and higher amplitudes of ripples and SWs than single SWR (Fig. $7 A, B$ ). Furthermore, the data demonstrate that previous learning experience changes the properties of SWRs, independent of their temporal relationship to DSW: the amplitude of ripples and SWs were significantly increased after fear conditioning (Fig. 7A), as were ripple frequencies and ripple amplitudes after spatial learning (Fig. $7 B$ ). Moreover, post hoc analyses showed that ripple frequency in the fear conditioning test (Fig. 7A, left panel) and SW amplitude in the spatial learning test (Fig. $7 B$, right panel) were significantly increased during the sleep phase after learning for the fraction of SWRs paired with DSWs, but not for single SWRs.

Although we saw an increase in ripple occurrence rate just after spatial learning, there were stronger SWRs after both spatial learning and fear conditioning, mediated by two learning-dependent effects. On the one hand, the fraction of SWRs that co-occured with DSWs increased, leading to a higher number of stronger SWRs. On the other hand, learning experiences increased the magnitude of the tested SWR properties (ripple frequency and amplitude, and SW amplitude) for both single SWRs and SWRs paired with DSWs. The observation that the more consistently observed learning-induced changes were on SWRs paired with DSWs suggests that this subset of SWRs might be more strongly affected by learning than SWRs not paired with DSWs.

We next tested whether there was an effect of previous learning experience on SWR, DSW, or DS2-associated MUA CCGs in CA1p or DG-GCL using a three-way ANOVA for repeated measures with "event type" (SWR, DSW, and DS2), "sleep phase" (before and after the learning trial), and "time bin" relative to the event (-700-700 ms, 100-ms bins) as within groups factors.
For the MUA in CA1p or DG-GCL associated with the fear conditioning test, there was no effect of sleep phase, or of the interactions between sleep phase and the other factors, indicating that MUA was not altered by the learning trial in this behavioral paradigm (Fig. $7 C$ ). In contrast, for the spatial learning task, there was a significant effect of the interaction between sleep phase, event type, and time bin on MUA in the CAlp $\left(F_{(28,112)}=\right.$ $2.01 ; p<0.01)$. Post hoc analyses showed that CA1p MUA synchrony was increased after the spatial learning trial as compared with the sleep epochs before the behavioral test during the 100ms CCG time bin around SWRs and DSWs but not during DS2 (Fig. 7D, top). For DG-GCL MUA, we found a significant effect of the interaction between sleep phase and time bin $\left(F_{(14,56)}=\right.$ 4.22; $p<0.0001)$ but no effect of the interaction between sleep phase, event type, and time bin $\left(F_{(28,112)}=1.06 ; p=0.3922\right)$. The overall MUA rates in CA1p or DG-GCL were not significantly different between pre-task and post-task sleep phases (data not shown). Post hoc tests indicated that DG-GCL MUA, comparable to MUA in CA1p, only showed learning-associated increases during the 100-ms CCG time bin around SWRs and DSWs, along with the -150 - to -50 -ms bin for SWRs, but not in any bin during DS2s (Fig. 7D, bottom). This ramp of DG GCL MUA in the preceding $-250 \mathrm{~ms}$ to an SWR parallels the slower intracellular membrane voltage ramps observed in DG GCL neurons before ripples during low-arousal states (Hulse et al., 2017). Altogether, our results suggest that, during post-experience sleep, CA3 output to dorsal CA1 and DG, but not EC output to the $\mathrm{DG}$, is modulated by spatial learning.

\section{Discussion}

In this study, we aimed at identifying experience-modulated LFP patterns during NREM sleep in the DG of chronically implanted mice. Cluster analysis of the current sinks and sources across the DG layers during baseline sleep reliably differentiated between the previously described DS2 and novel DSW events in all of our recordings. After identifying DSWs, we hypothesized that they could be an electrophysiological correlate of ongoing memory consolidation processes in the DG.

\section{Cluster analysis of DG CSD profiles differentiates between DS and DSWs}

We developed a detection and classification method allowing us to differentiate between DS2s and DSWs without bias from the LFP activity of non-DG hippocampal regions. In contrast to previous studies, we used a lower amplitude threshold for event detection and did not exclude wide-waveform events, which resulted in a higher detection frequency of DS2 during NREM sleep of $\sim 0.5-1 \mathrm{~Hz}$ compared with $\sim 0.25 \mathrm{~Hz}$ (Bragin et al., 1995), $\sim 0.5 \mathrm{~Hz}$ (Headley et al., 2017), or $\sim 0.04 \mathrm{~Hz}$ (Nokia et al., 2017). Our DS2 cluster may include events triggered by cortical down-up state transitions that evoke similar CSD depth profiles in DG, which can also precede SWRs (Battaglia et al., 2004; Isomura et al., 2006; Mölle et al., 2006; Sullivan et al., 2011). The original description of DS2 in rats showed the absence of SWR activity within a 200-ms window around the DS2 (Bragin et al., 1995). In mice, we found that MUA in CA1p increased roughly $100 \mathrm{~ms}$ after a DS2. DSWs, by comparison, occurred in tandem with rapidly decaying MUA peaks, which were strongest in DG GCL, but were also seen in CA1p.

\section{DSWs are strongly correlated to CA1 SWR}

The DG receives excitatory input from the EC and CA3. Both inputs can evoke large amplitude waves during NREM sleep: EC 
induces DS2s, and CA3 induces the DG potentials seen during SWRs. Unlike previous studies showing SW-like potentials in the DG (Ylinen et al., 1995; Penttonen et al., 1997; Sullivan et al., 2011), we identified DSWs that also occurred independently of local SWR activity in CA1. The coupling of DSWs to a large fraction of SWRs and the parallel increase in CA1p and DGGCL MUA suggest that both DSWs and SWRs may be induced by a cooperative discharge of CA3 pyramidal cells. We therefore hypothesize that DSWs are induced by CA3 feedback projections.

\section{DSW-paired SWRs are stronger than single SWRs}

We found that a large fraction $(\sim 30 \%)$ of DSWs were paired with SWRs and vice versa $(\sim 20 \%)$. The SWRs that were paired with DSWs were larger in amplitude, the ripples exhibited a higher oscillatory frequency, and MUA in CA1p and DG was higher than during unpaired SWRs. SWs in the CA1 stratum radiatum are induced by extensive axon (Schaffer) collateral activation of CA3 pyramidal neurons that exerts spatially widespread effects on CA1 neurons. Ripples can emerge locally, at single or multiple locations in CA1, by the interaction of CA1 pyramidal cells and interneurons (Ylinen et al., 1995; Stark et al., 2014). Ripples with a higher amplitude occur synchronously over larger distances, or travel along the longitudinal axis of the CA1p region, whereas low-magnitude ripples remain localized (Patel et al., 2013). Given these findings, our results suggest that DSWs are preferentially paired with SWRs that involve a larger spatial extent of the CA1 region. However, this hypothesis remains to be tested in a dataset involving multiple recording sites along the dorsoventral axis of the hippocampus.

During single SWRs, we found current sinks in the DG IML and an increase in DG MUA (data not shown; sink amplitude of single SWR was similar to average SWR in Fig. $3 A, E$ ), which suggests that low-amplitude SWRs may co-occur with low-amplitude DSWs that fell below our detection threshold.

Previous studies showed that interference with SWRs by stimulation of the vHC impairs spatial memory consolidation (Girardeau et al., 2009, 2014; Ego-Stengel and Wilson, 2010). Stimulation of the vHC also induced population spikes in the DG and was sufficient to impair associative learning when targeted at DS (Nokia et al., 2017). Previous findings showed a modest OML/IML source/sink pair across the DG during CA1 ripples (Sullivan et al., 2011) as would arise from a fraction of SWRs co-occurring with DSWs, as is seen in our data. It is thus possible that the spatial learning impairment associated with vHC stimulation might not only result from SWR disruption in CA1, but could also be due to the induction of unselective coactivation of CA1, CA3, and DG, thereby disrupting the temporal coordination between SWRs and DSWs. Along these lines, the selective inhibition of CA1 activity during ripples did not significantly alter place field structure (Kovács et al., 2016).

\section{Rapid fall in DG MUA after DSWs is consistent with inhibition in the DG}

In previous studies, an increase in DGC activity was found during spontaneous SWRs (Penttonen et al., 1997), which was mediated by feed-back activation of the DG. We found that MUA in the DG and in the CA1p region increased before the trough of a DSW and rapidly decreased thereafter. As has been observed in CAlp (Hulse et al., 2016), the rapid decrease of activity in DGGCL could be because of local feed-forward and feed-back inhibition. The activity of several types of interneurons in the DG is differentially modulated by the stimulation of the DG afferents, or during spontaneous DG LFP patterns. The axons of these classes of interneurons project to different subcellular locations of the DGCs (Hsu et al., 2016; Szabo et al., 2017). Furthermore, the activities of several interneuron populations, including those expressing parvalbumin, somatostatin, or cannabinoid receptor 1, show different temporal relationships to SWRs, which generate spatially and temporally distributed inhibition during SWRs (Szabo et al., 2017). By recruitment of these interneuronal networks, DSWs could be involved in a closed-loop circuit in which CA3 induces feed-back activation of DG-GCs and DG interneurons, which subsequently mediate feed-forward inhibition of CA3. Moreover, interneurons with projections to the OML and MML could be recruited during DSWs to shunt EC input by dendritic inhibition. This mechanism could serve to gate concurrent CA3 and EC input, thereby providing a temporal window during DSWs in which the DGCs selectively respond to information received by the CA3 backprojection, thus separating it from EC input.

\section{DSWs may integrate information along the longitudinal axis of the hippocampus}

It is known from experiments in rats that the SWR occurrence rate and ripple amplitude are modulated by prior experience (Eschenko et al., 2008; Ramadan et al., 2009; Girardeau et al., 2014). Previous anatomic studies have shown that the hippocampus consists of subregions along the longitudinal axis. In a simplified dichotomous view, the $\mathrm{dHC}$ processes spatial information, while the vHC mediates emotional and anxiety-related behaviors (Ranganath and Ritchey, 2012; Kheirbek et al., 2013; Strange et al., 2014). SWR propagation, too, was shown to be isolated among dorsal/intermediate parts and ventral CA1 (Patel et al., 2013). Recent work on the Nucleus accumbens (NAc) to HC connectivity has uncovered that SWR-related communication in the dHC-NAc and vHC-NAc pathways occurred at different times and that SWRs in $\mathrm{dHC}$, but not in vHC, activated NAc neurons encoding spatial and reward reward information (Sosa et al., 2020). These findings suggest that memory consolidation processes specifically modulate SWR occurrence in hippocampal subregions. Our data from mice support this notion because we show that the generation of SWRs, as well as MUA during SWRs and DSWs, is modulated by spatial learning but not by fear conditioning. In contrast, DSW occurrence rates increased after both learning paradigms and, thus, they appear to be a novel, sensitive measure for the characterization of general learninginduced changes in $\mathrm{dHC}$.

Hilar activity has been hypothesized to be generated by the CA3 backprojection (Ylinen et al., 1995; Penttonen et al., 1997), which consists of direct projections of ventral CA3 to the IML of the DG, as well as of relayed projections from CA3 via hilar mossy cells, which also project to the IML of the DG (Li et al., 1994; Scharfman, 2007). Hilar mossy cells are activated during SWRs (Swaminathan et al., 2018), project to $\sim 75 \%$ of the longitudinal axis of the hippocampus (Amaral et al., 2007) and, therefore, could elicit DSWs at a distance. The increased occurrence of DSWs after fear conditioning and spatial learning trials could be explained by the widespread projections of hilar mossy cells along the longitudinal axis of the hippocampus, which may lead to a global activation of DGCs during DSWs compared with the localized occurrence of SWRs. DSWs may thereby provide a framework for the integration of information of different qualities, encoded during awake states and processed separately in ventral and dorsal parts of the hippocampus. During DSWs, the information could simultaneously be reactivated by auto- 
associative networks in CA3 and via feedback activation of the DG. However, to test this hypothesis, simultaneous recordings from $\mathrm{dHC}$ and $\mathrm{vHC}$ regions will be needed to elucidate the spatiotemporal characteristics of the CA3-dependent concurrent activation of CA1 and DG. In summary, DSWs are strongly related to SWRs, which are well described as biomarkers of memory consolidation. When recording at a single electrode shank, the quantification of DSWs may be a more sensitive measure of ongoing memory consolidation processes in the hippocampus than that of SWRs recorded at the same location. We hypothesize that DSWs may be a signature of coordinated information transfer between widely spaced hippocampal subregions. Although our data clearly show that DSW activity is affected by previous experience, further functional studies are required to test the hypothesis that the learning-induced DSW activity is required for long-term memory consolidation. We nonetheless present DSWs as a complementary experience-driven hippocampal activity pattern visible during memory consolidation processes in mice.

\section{References}

Abel T, Havekes R, Saletin JM, Walker MP (2013) Sleep, plasticity and memory from molecules to whole-brain networks. Curr Biol 23:R774-R788.

Allen Institute for Brain Science (2004) Allen mouse brain atlas. Available at http://atlas.brain-map.org.

Amaral DG, Scharfman HE, Lavenex P (2007) The dentate gyrus: fundamental neuroanatomical organization (dentate gyrus for dummies). Prog Brain Res 163:3-22.

Battaglia FP, Sutherland GR, McNaughton BL (2004) Hippocampal sharp wave bursts coincide with neocortical "up-state" transitions. Learn Mem 11:697-704.

Bokil H, Andrews P, Kulkarni JE, Mehta S, Mitra PP (2010) Chronux: a platform for analyzing neural signals. J Neurosci Methods 192:146-151.

Bragin A, Jandó G, Nádasdy Z, van Landeghem M, Buzsáki G (1995) Dentate EEG spikes and associated interneuronal population bursts in the hippocampal hilar region of the rat. J Neurophysiol 73:1691-1705.

Buzsáki G (1986) Hippocampal sharp waves: their origin and significance. Brain Res 398:242-252.

Buzsáki G, Buhl DL, Harris KD, Csicsvari J, Czéh B, Morozov A (2003) Hippocampal network patterns of activity in the mouse. Neuroscience 116:201-211.

Dimitriadis G, Neto J, Kampff A (2018) t-SNE visualization of large-scale neural recordings. Neural Comput 30:1750-1774.

Ego-Stengel V, Wilson MA (2010) Disruption of ripple-associated hippocampal activity during rest impairs spatial learning in the rat. Hippocampus 20:1-10.

Eschenko O, Ramadan W, Mölle M, Born J, Sara SJ (2008) Sustained increase in hippocampal sharp-wave ripple activity during slow-wave sleep after learning. Learn Mem 15:222-228.

Ester M, Kriegel HP, Sander J, Xu X (1996) A density-based algorithm for discovering clusters in large spatial databases with noise. Proc 2nd Int Conf Knowl Discov Data Min 226-231. Available at https://www.aaai. org/Papers/KDD/1996/KDD96-037.pdf.

Fellini L, Morellini F (2013) Mice create what-where-when hippocampus-dependent memories of unique experiences. J Neurosci 33:1038-1043.

Girardeau G, Benchenane K, Wiener SI, Buzsáki G, Zugaro MB (2009) Selective suppression of hippocampal ripples impairs spatial memory. Nat Neurosci 12:1222-1223.

Girardeau G, Cei A, Zugaro M (2014) Learning-induced plasticity regulates hippocampal sharp wave-ripple drive. J Neurosci 34:5176-5183.

Hazan L, Zugaro M, Buzsáki G (2006) Klusters, NeuroScope, NDManager: a free software suite for neurophysiological data processing and visualization. J Neurosci Methods 155:207-216.

Headley DB, Kanta V, Paré D (2017) Intra- and interregional cortical interactions related to sharp-wave ripples and dentate spikes. J Neurophysiol 117:556-565.

Hsu T, Lee C, Tai M, Lien C (2016) Differential recruitment of dentate gyrus interneuron types by commissural versus perforant pathways. Cereb Cortex 26:2715-2727.
Hulse BK, Moreaux LC, Lubenov EV, Siapas AG (2016) Membrane potential dynamics of CA1 pyramidal neurons during hippocampal ripples in awake mice. Neuron 89:800-813.

Hulse BK, Lubenov EV, Siapas AG (2017) Brain state dependence of hippocampal subthreshold activity in awake mice. Cell Rep 18:136-147.

Isomura Y, Sirota A, Ozen S, Montgomery S, Mizuseki K, Henze DA, Buzsáki G (2006) Integration and segregation of activity in entorhinalhippocampal subregions by neocortical slow oscillations. Neuron 52:871882.

Kadir SN, Goodman DFM, Harris KD (2014) High-dimensional cluster analysis with the masked EM algorithm. Neural Comput 26:2379-2394.

Kheirbek MA, Drew LJ, Burghardt NS, Costantini DO, Tannenholz L, Ahmari SE, Zeng H, Fenton AA, Hen R (2013) Article differential control of learning and anxiety along the dorsoventral axis of the dentate gyrus. Neuron 77:955-968.

Kovács KA, O’Neill J, Schoenenberger P, Penttonen M, Ranguel Guerrero DK, Csicsvari J (2016) Optogenetically blocking sharp wave ripple events in sleep does not interfere with the formation of stable spatial representation in the CA1 area of the hippocampus. PLoS One 11:e0164675.

Lasztóczi B, Klausberger T (2017) Distinct gamma oscillations in the distal dendritic fields of the dentate gyrus and the CA1 area of mouse hippocampus. Brain Struct Funct 222:3355-3365.

Lee AK, Wilson MA (2002) Memory of sequential experience in the hippocampus during slow wave sleep. Neuron 36:1183-1194.

Lein ES, Hawrylycz MJ, Ao N, Ayres M, Bensinger A, Bernard A, Boe AF, Boguski MS, Brockway KS, Byrnes EJ, Chen L, Chen L, Chen TM, Chin MC, Chong J, Crook BE, Czaplinska A, Dang CN, Datta S, Dee NR, et al. (2007) Genome-wide atlas of gene expression in the adult mouse brain. Nature 445:168-176.

Lensu S, Waselius T, Penttonen M, Nokia MS (2019) Dentate spikes and learning: disrupting hippocampal function during memory consolidation can improve pattern separation. J Neurophysiol 121:131-139.

Leutgeb JK, Leutgeb S, Moser MB, Moser EI (2007) Pattern separation in the dentate gyrus and CA3 of the hippocampus. Science 315:961-966.

Li XG, Somogyi P, Ylinen A, Buzsáki G (1994) The hippocampal CA3 network: an in vivo intracellularly labeling study. J Comp Neurol 339:181208.

Maingret N, Girardeau G, Todorova R, Goutierre M, Zugaro M (2016) Hippocampo-cortical coupling mediates memory consolidation during sleep. Nat Neurosci 19:959-964.

Mölle M, Yeshenko O, Marshall L, Sara SJ, Born J (2006) Hippocampal sharp wave-ripples linked to slow oscillations in rat slow-wave sleep. J Neurophysiol 96:62-70.

Nakazawa K, Quirk MC, Chitwood RA, Watanabe M, Yeckel MF, Sun LD, Kato A, Carr CA, Johnston D, Wilson MA, Tonegawa S (2002) Requirement for hippocampal CA3 NMDA receptors in associative memory recall. Science 297:211-218.

Nokia MS, Gureviciene I, Waselius T, Tanila H, Penttonen M (2017) Hippocampal electrical stimulation disrupts associative learning when targeted at dentate spikes. J Physiol 595:4961-4971.

O’Reilly RC, McClelland JL (1994) Hippocampal conjunctive encoding, storage, and recall: avoiding a trade-off. Hippocampus 4:661-682.

Patel J, Schomburg EW, Berényi A, Fujisawa S, Buzsáki G (2013) Local generation and propagation of ripples along the septotemporal axis of the hippocampus. J Neurosci 33:17029-17041.

Paxinos G, Franklin KBJ (2007) The mouse brain in stereotaxic coordinates. Ed 3. San Diego: Academic Press.

Penttonen M, Kamondi A, Sik A, Acsády L, Buzsáki G (1997) Feed-forward and feed-back activation of the dentate gyrus in vivo during dentate spikes and sharp wave bursts. Hippocampus 7:437-450.

Perrin F, Reggers J, Phillips C, Degueldre C, Fiore GD, Peigneux P, Laureys S, Fuchs S, Collette F, Del Fiore G, Aerts J, Luxen A, Maquet P (2004) Are spatial memories strengthened in the human hippocampus during slow wave sleep? Neuron 44:535-545.

Pettersen KH, Devor A, Ulbert I, Dale AM, Einevoll GT (2006) Currentsource density estimation based on inversion of electrostatic forward solution: effects of finite extent of neuronal activity and conductivity discontinuities. J Neurosci Methods 154:116-133.

Ramadan W, Eschenko O, Sara SJ (2009) Hippocampal sharp wave/ripples during sleep for consolidation of associative memory. PLoS One 4:e6697.

Ranganath C, Ritchey M (2012) Two cortical systems for memory-guided behaviour. Nat Rev Neurosci 13:713-726. 
Scharfman HE (2007) The CA3 "backprojection" to the dentate gyrus. Prog Brain Res 163:627-637.

Shi Y, Grieco SF, Holmes TC, Xu X (2019) Development of local circuit connections to hilar mossy cells in the mouse dentate gyrus. eNeuro 6: ENEURO.0370-18.2019.

Sosa M, Joo HR, Frank LM (2020) Dorsal and ventral hippocampal sharpwave ripples activate distinct nucleus accumbens networks. Neuron 105:725-741.e8.

Stark E, Roux L, Eichler R, Senzai Y, Royer S, Buzsáki G (2014) Pyramidal cell-interneuron interactions underlie hippocampal ripple oscillations. Neuron 83:467-480

Stickgold R (2005) Sleep dependent memory consolidation. Nature 437:1272-1278.

Strange BA, Witter MP, Lein ES, Moser EI (2014) Functional organization of the hippocampal longitudinal axis. Nat Rev Neurosci 15:655-669.

Sullivan D, Csicsvari J, Mizuseki K, Montgomery S, Diba K, Buzsáki G, Buzsáki G (2011) Relationships between hippocampal sharp waves, ripples, and fast gamma oscillation: influence of dentate and entorhinal cortical activity. J Neurosci 31:8605-8616.
Swaminathan A, Wichert I, Schmitz D, Maier N (2018) Involvement of mossy cells in sharp wave-ripple activity in vitro. Cell Rep 23:25412549.

Szabo GG, Du X, Oijala M, Varga C, Parent JM, Soltesz I (2017) Extended interneuronal network of the dentate gyrus. Cell Rep 20:12621268.

Treves A, Rolls ET (1992) Computational constraints suggest the need for two. Hippocampus 2:189-200.

van der Maaten L (2014) Accelerating t-SNE using tree-based algorithms. J Mach Learn Res 15:3221-3245.

Wilson MA, McNaughton BL (1994) Reactivation of hippocampal ensemble memories during sleep. Science 265:676-617.

Ylinen A, Bragin A, Nádasdy Z, Jandó G, Szabó I, Sik A, Buzsáki G (1995) Sharp wave-associated high-frequency oscillation $(200 \mathrm{~Hz})$ in the intact hippocampus: network and intracellular mechanisms. J Neurosci 15:3046.

Zhang H, Fell J, Axmacher N (2018) Electrophysiological mechanisms of human memory consolidation. Nat Commun 9. 